\title{
Interplay between Heavy Fermions and Crystal Field Excitation in Kondo Lattices. Low-Temperature Thermodynamics and Inelastic Neutron Scattering Spectra of CeNiSn
}

\author{
Yu. Kagan, K.A. Kikoin and A.S. Mishchenko \\ Kurchatov Institute, Moscow 123182, Russia
}

\begin{abstract}
The microscopic theory of interaction between the heavy fermions and the crystal field excitations in Kondo lattices is presented. It is shown that the heavy-fermion spectrum scaled by the Kondo temperature $T_{K}$ can be modified by the crystal field excitations with the energy $\Delta_{C F}$ provided the inequality $\Delta_{C F}<T_{K}$ is realized. On the base of general description of excitation spectrum the detailed qualitative and quantitative explanation of anisotropic inelastic neutron scattering spectra and low-temperature specific heat of orthorhombic CeNiSn is given. The theory resolves the apparent contradiction between the metallic conductivity and the gap-wise behavior of thermodynamic properties and spin response of CeNiSn at low temperatures.
\end{abstract}

Keywords: Kondo effect, crystal fields, magnetic correlations

\section{Contact address:}

\author{
A.S.Mishchenko \\ RRC Kurchatov Institute, Moscow 123182, Russia \\ tel: (7)(095)1969148; \\ fax: (7)(095)1965973; \\ e-mail: andry@kurm.polyn.kiae.su
}




\section{Introduction}

Although the heavy fermion (HF) behavior of the rare earth- and actinide-based intermetallides is related to the localized electrons in the $4 \mathrm{f}$ - and $5 \mathrm{f}$-shells of $\mathrm{Ce}$ and $\mathrm{U}$, the influence of the crystal field (CF) splitting of the f-levels is inessential, as a rule, for the low-energy excitations in these systems, since the inequality $\Delta_{C F} \gg T^{*}$ is valid for most of these compounds [1]. Here $T^{*} \sim T_{K}$ is the temperature which characterizes the energy scale of the HF spectrum. The only role of this interaction is to form the ground state Kramers doublet which is the eventual source of the low-energy excitations with extremely high density of states. However, the picture changes radically when this inequality is violated. One can expect that in case of $\Delta_{C F}<T^{*}$ the inevitable interplay between the local CF excitations and itinerant HFs should result in appearance of the fine structure in the low-energy spectrum which should be characterized by at least two additional energy parameters, i.e., the position of the CF level and the magnitude of intermixing. This interplay should result in remarkable changes in the spectral and thermodynamic properties of the system in the temperature interval $0<T<\Delta_{C F}$, although it is clear that the characteristic HF behavior of the system should persist at $T \rightarrow$ 0: the Fermi liquid-like temperature dependences for the specific heat, $C(T) \sim T$, electrical resistivity, $\rho(T)-\rho(0) \sim T^{2}$, NMR relaxation rate, $T_{1} T=$ const, etc., should be observed, although the coefficients in these laws should be sensitive not only to $T^{*}$ but also to these new parameters. On the other hand, the hybridization of the HF and CF excitation can result, as it frequently occurs, in appearance of the psedogap in the energy spectrum, although this pseudogap should be characterized mainly by the crystal field and mixing parameters.

The idea of applying these considerations to description of unusual properties of CeNiSn was proposed in [2]. It was shown that one of the necessary preconditions for realization of this mechanism is the specific picture of CF level splitting: it was supposed that two lowest Kramers doublet of $\mathrm{Ce}\left(\mathrm{f}^{1}\right)$ configuration are separated by a small energy gap

$$
\Delta_{C F}<T^{*}
$$

while the distance between the ground state and the second excited level is much larger, $\Delta_{C F}^{\prime} \gg$ $\Delta_{C F}$. The characteristic HF energy $T^{*}$ is estimated as 40 to $80 \mathrm{~K}$ from various experimental data for CeNiSn [3]. Later on, the indirect measurements by Alekseev et al [4] confirmed the assumption (西). In that paper the crystal field was measured on the $\mathrm{Nd}$ ion in $\mathrm{Nd}_{0.3} \mathrm{La}_{0.7} \mathrm{Ni}$ crystal which is equivalent to CeNiSn from the point of view of RE crystalline environment and lattice spacings. The crystal field acting at the RE site was restored from measured CF splitting of $\mathrm{Nd}$ f-level and then recalculated for the case of Ce ion with the same parameters of equivalent $\mathrm{CF}$ Hamiltonian in approximation of purely electrostatic field. The CF splitting energies were estimated as $\Delta_{C F} \approx 4.4 \mathrm{meV}$ and $\Delta_{C F}^{\prime} \approx 14.0 \mathrm{meV}$ which is consistent with the assumption of ref. [2] and the inequality (11).

Another experimental result crucial for the above general consideration was the observation of Fermi-liquid behavior at low enough temperature in good quality samples of CeNiSn and CeRhSb. It was found that these samples demonstrate the metallic $T^{2}$-dependence of electrical resistivity at $T<7 \mathrm{~K}$ [5], the NMR relaxation rate returns to Korringa law at $T<1 \mathrm{~K}$ [6], and the limiting value of the Sommerfeld coefficient $\gamma$ in the linear- $T$ term of $C(T)$ is $\gamma=40 \mathrm{~mJ} / \mathrm{K}^{2} \mathrm{~mol}$ at $0.03 \mathrm{~K} \mathrm{[5]}$. This value is noticeably lower than that predicted by the high-temperature estimations of $T^{*}$. These results seemed to be striking, since the semiconductor-like rise of the resistivity and $T^{3}$ dependence of $1 / T_{1}$ was observed earlier on the less perfect samples [3]. 
The most challenging experimental observations on CeNiSn are the inelastic magnetic neutron scattering spectra which demonstrate extremely complicated $(\mathbf{Q}, \omega)$-dependent structures with quasi gap behavior for some directions of $\mathbf{Q}$ [0, 8, 9, 10]. All these features disappear completely at $\mathrm{T}=25 \mathrm{~K}$. This picture is inexplicable in terms of standard notions of intersite spin fluctuations. At the same time, these data are highly informative from the point of view of the structure of low energy spin excitations, so the adequate interpretation of the neutron scattering data is the most effective criterion for selecting out of various theoretical interpretations.

In the present paper the spectral properties of CeNiSn are analyzed on the base of the ideas formulated in [2] and applied for the quantitative explanation of low temperature thermodynamics in [2, 11]. We apply these ideas to the spin liquid which is formed in Kondo lattices, provided the magnetic order does not appear at low temperature. We describe this spin liquid in terms of resonating valence bond (RVB) excitations similar to those introduced by P. Anderson and used later in description of nearly magnetic state in $2 \mathrm{D} \mathrm{Cu}-\mathrm{O}$ planes of high- $\mathrm{T}_{c}$ materials [12]. In this case the characteristic properties of HF liquid are determined by the subsystem of spin excitations (spinons) which obey Fermi statistics at low temperature. The stabilization mechanism for the RVB state in 3D Kondo lattices was offered in 13. We modify the theory of spin liquid for the case of $\Delta_{C F}<T_{K}$, calculate the spectrum of RVB excitations for a special case of orthorhombic CeNiSn lattice and give a detailed comparison of calculated neutron scattering cross section with the experimental results of [9, 10]. The quasi 2D character of spin excitations discovered in these experiments is essentially used in our calculations. Quite reasonable agreement between the theoretical curves and experimental constant- $\mathbf{Q}$ and constant- $E$ scans seems encouraging. To make an additional test of adequacy of the theory, we calculate also the low-temperature specific heat of CeNiSn within the same set of model parameters.

\section{Degenerate spin liquid in a crystal field}

The theory of HF systems starts with the Anderson lattice Hamiltonian. In a special case of $\mathrm{Ce}^{(3+)}\left(f^{1}\right)$ ions in a crystal field this Hamiltonian is usually written as

$$
H=H_{f}+H_{c}+H_{c f}+H^{\prime}
$$

Here $H_{f}$ describes the Ce ions in a crystal field,

$$
H_{f}=\sum_{\mathbf{i}, \Lambda} E_{\Gamma} f_{\mathbf{i} \Lambda}^{\dagger} f_{\mathbf{i} \Lambda}+\frac{U}{2} \sum_{\mathbf{i}, \Lambda \neq \Lambda^{\prime}} f_{\mathbf{i} \Lambda}^{\dagger} f_{\mathbf{i} \Lambda} f_{\mathbf{i} \Lambda^{\prime}}^{\dagger} f_{\mathbf{i} \Lambda^{\prime}}
$$

$f_{\mathbf{i} \Lambda}^{\dagger}$ is the creation operator of the f-electron in a state $\Lambda=\Gamma \nu$ in a site $\mathbf{i}$, where $\nu$ is the row of the irreducible representation $\Gamma$ of the crystal point group, $E_{\Gamma}$ denotes the energy of the f-level in the electrostatic crystal field, and $U$ stands for the Coulomb repulsion between the f-electrons in the same site. The f-ions are immersed in a Fermi-sea of conduction electrons which are described by the Hamiltonian

$$
H_{c}=\sum_{k, \Lambda} \epsilon_{k \Gamma} c_{k \Lambda}^{\dagger} c_{k \Lambda}
$$

The third term in the Hamiltonian (2) describes the hybridization between $\mathrm{f}$ - and conduction electrons,

$$
H_{c f}=\sum_{k k^{\prime}} \sum_{\mathbf{i}} \sum_{\Lambda}\left(V_{k \Lambda}^{\mathbf{i}} f_{\mathbf{i} \Lambda}^{\dagger} c_{k \Lambda}+H . c .\right)
$$


The so called Coqblin-Cornut (CC) approximation [14, 15] which represents the Bloch functions by their partial waves $c_{k \Lambda}^{\dagger}$, and takes into account only the diagonal in $\Lambda$ hybridization matrix elements $V_{k \Lambda}^{\mathbf{i}}=\langle\mathbf{k} \Lambda|V| \mathbf{i} \Lambda\rangle$ is used. All the one-electron processes beyond this approximation are collected in the last term $H^{\prime}$ in the Hamiltonian (2).

It is widely believed that the $\mathrm{s}=1 / 2$ approximation for the total moment $j$ of $\mathrm{Ce}\left(\mathrm{f}^{1}\right)$ ion works well in the Anderson lattice Hamiltonian because the CF splitting of the sextet $\mathrm{j}=5 / 2$ usually results in a simple Kramers doublet ground state, and the CF splitting exceeds essentially the characteristic interaction energy $T_{K}$. This procedure seems to be reasonable when one deals with the ground state and the low-energy excitations with characteristic frequencies $\hbar \omega<\Delta_{C F}$. Since our task is inclusion of CF excitations in the general picture of spin liquid behavior, we should begin with generalization of $\mathrm{s}=1 / 2$ theory for the case of realistic crystal field scheme.

The Kondo lattice deals with the well localized f-electrons for which the inequality $V_{\mathbf{k} \Lambda} \ll$ $\epsilon_{F}-E_{f}$, where $E_{f}$ is the energy level of free $\mathrm{Ce}\left(\mathrm{f}^{1}\right)$ ion, is assumed to be valid, and the Hubbard parameter $U$ is taken large enough to suppress the doubly occupied f-states of this ion. In this case the canonical transformation eliminating the hybridization interaction from the Hamiltonian (2) can be done (see [15]), and the effective Hamiltonian projected to the subspace of homopolar states with $n_{f}=1$ can be written as

$$
H^{(C C)}=H_{f}+H_{c}+H_{e x}+H_{h}
$$

where

$$
H_{e x}=\sum_{k k^{\prime}} \sum_{\mathbf{i}} \sum_{\Lambda, \Lambda^{\prime}} J_{\mathbf{i}}^{\Lambda \Lambda^{\prime}}\left(k, k^{\prime}\right) f_{\mathbf{i} \Lambda}^{\dagger} f_{\mathbf{i} \Lambda^{\prime}} c_{k^{\prime} \Lambda^{\prime}}^{+} c_{k \Lambda}
$$

describes the effective sf-exchange interaction, and

$$
H_{h}=-\sum_{k} \sum_{\mathbf{i}} \sum_{\Lambda} J_{\mathbf{i}}^{\Lambda \Lambda}(k, k) f_{\mathbf{i} \Lambda}^{\dagger} f_{\mathbf{i} \Lambda}
$$

corresponds to effective covalent contribution to the one-site CF splitting due to virtual sftransitions. Here

$$
J_{\mathbf{i}}^{\Lambda \Lambda^{\prime}}\left(k, k^{\prime}\right)=\frac{V_{k \Lambda}^{\mathbf{i} *} V_{k^{\prime} \Lambda^{\prime}}^{\mathbf{i}}}{\epsilon_{k}-E_{f}} .
$$

We assume for the sake of simplicity that the conduction electrons are degenerate in $\Lambda$ near the electron Fermi surface and neglect the CF splitting of the f-level in the denominator of effective sf-exchange integral.

We are interested in the case of $n_{f}=1$ when the charge fluctuations in the f-channel are completely suppressed. Then, according to the scenario of [13, 16] the neutral spin liquid should arise at some temperature $T^{*} \sim T_{K}$ instead of antiferromagnetic or Kondo-singlet state offered by the standard Doniach's dichotomy. And, as a result, the spin liquid excitations together with the low-energy electrons turn out to be responsible for the low-temperature thermodynamics of the Kondo lattice. Now we incorporate the CF excitations in this picture. The "fast" electrons with characteristic energies $\hbar \omega>T_{K}$ are responsible for formation of the spin-spin correlation at $T>T_{K}$ (the Kondo temperature is defined as $T_{K} \approx \epsilon_{f} \exp (-1 / 2 \alpha)$, where $\alpha=J_{s f} \mathcal{N}\left(\epsilon_{F}\right)$, $J_{s f}=\left|V_{k_{F}}\right|^{2} /\left(\epsilon_{F}-E_{f}\right)$, and $\mathcal{N}\left(\epsilon_{F}\right)$ is the electron density of states at Fermi level). These fast electrons can be integrated out, and the effective Hamiltonian for the spin degrees of freedom can be written in the following form.

$$
H^{s}=H_{f}+H_{h}^{\prime}+H_{R K K Y}^{(g)}+H_{R K K Y}^{(n d)}
$$




$$
\begin{aligned}
& H_{R K K Y}^{(g)}=\sum_{\mathbf{i} \mathbf{i}^{\prime}}^{\mathbf{i} \neq \mathbf{i}^{\prime}} \sum_{\nu \nu^{\prime}} I_{\mathbf{i i}^{\prime}}^{G G} f_{\mathbf{i} G \nu}^{\dagger} f_{\mathbf{i} G \nu^{\prime}} f_{\mathbf{i}^{\prime} G \nu^{\prime}}^{\dagger} f_{\mathbf{i}^{\prime} G \nu}, \\
& H_{R K K Y}^{(n d)}=\sum_{\mathbf{i} \neq \mathbf{i}^{\prime}} \sum_{E \nu \nu^{\prime}} I_{\mathbf{i i}^{\prime}}^{G E} f_{\mathbf{i} G \nu}^{\dagger} f_{\mathbf{i} E \nu^{\prime}} f_{\mathbf{i}^{\prime} E \nu^{\prime}}^{\dagger} f_{\mathbf{i}^{\prime} G \nu}
\end{aligned}
$$

Here the notations $\Lambda=G \nu$ and $\Lambda=E \nu$ are used for the ground state and excited states, respectively, $H_{h}^{\prime}$ includes the scattering correction to the $\mathrm{CF}$ level renormalization due to the interaction $H_{e x}$,

$$
I_{\mathbf{i i}^{\prime}}^{G G} \sim \alpha^{2} \Phi_{G}\left(\mathbf{k}_{F}, \mathbf{R}_{i}-\mathbf{R}_{i^{\prime}}\right) B_{G}(\theta, \phi) K(T)
$$

is the indirect exchange interaction which contains oscillating RKKY function $\Phi_{G}\left(\mathbf{k}_{F}, \mathbf{R}_{i}-\mathbf{R}_{i^{\prime}}\right)$, anisotropy factor $B_{G}(\theta, \phi)$ ( $\theta$ is the angle between the $z$-axis which is assumed to be the axis of magnetic quantization, and the line connecting the ions $\mathbf{R}_{i}$ and $\mathbf{R}_{i^{\prime}}$ and $\phi$ is the angle of rotation about $z$-axis) (see Appendix), and additional enhancement factor $K(T)$ due to the high-temperature one-site Kondo scattering [13]. $I_{\mathrm{ii}^{\prime}}^{G E}$ is the integral of the same type as that in eq. (13), with its own factors $B_{G E}$ and $\Phi_{G E}$, and $K_{G E}(T)=1$.

The uniform spin liquid state of RVB type in a standard $s=1 / 2$ Hesenberg model is described in terms of correlators [12],

$$
\Delta=\sum_{\sigma}\left\langle f_{\mathbf{i} \sigma}^{\dagger} f_{\mathbf{i}^{\prime} \sigma}\right\rangle, i \neq i^{\prime}
$$

It was shown in [13] that the weak-coupling Kondo interaction at $T>T_{K}$ favors the stabilization of RVB state against antiferromagnetic ordering because the Kondo scattering screens the local moment leaving intact the singlet RVB correlators (14). This stabilization "quenches" the Kondo scattering at temperatures $T \sim T^{*}>T_{K}$ and allows the description of spin liquid in terms of RVB correlation functions at $T<T_{K}$, since $T_{K}$ is no more a singular point of perturbation theory. Having in mind this stabilization mechanism we use the mean-field approximation for the uniform RVB state even at $T<T_{K}$. Although the interaction with "slow" conduction electrons and the influence of spin and gauge fluctuations can modify the properties of spin-fermions, we use this mean-field description as the basic approximation for studying the system with the CF excitations involved.

Thus, the correlator similar to (14) can be introduced for the ground state doublet $G \nu$ :

$$
\Delta^{G}=\sum_{\nu}\left\langle f_{\mathbf{i} G \nu}^{\dagger} f_{\mathbf{i}^{\prime} G \nu}\right\rangle, i \neq i^{\prime}
$$

The spin-fermion excitations which are described by the Hamiltonian (10) are constrained by the condition

$$
\sum_{\Lambda} f_{\mathbf{i} \Lambda}^{\dagger} f_{\mathbf{i} \Lambda}=1
$$

Important contributions to the interplay between the low-energy spinon excitations and the one-site CF excitations are connected with the interactions $H^{\prime}$ which appear in the Hamiltonian (2) beyond CC approximation. The origin of these interactions is the hybridization $\bar{V}_{k \Lambda}^{\mathrm{i} \Lambda^{\prime}}=$ $\left\langle\mathbf{i} \Lambda\left|V^{\prime}\right| k \Lambda^{\prime}\right\rangle$ where $V^{\prime}$ is the component of the crystal field which has the symmetry lower than that diagonalizing the energy terms $E_{\Gamma}$. When the integrals $\bar{V}_{k \Lambda}^{\mathrm{i} \Lambda^{\prime}}$ are included in the canonical 
transformation, additional effective exchange terms appear:

$$
H_{e x}^{\prime}=\sum_{k k^{\prime}} \sum_{\mathbf{i}, E, \nu \nu^{\prime} \nu^{\prime \prime}}\left[\frac{\bar{V}_{k G}^{\mathbf{i} E *} V_{k^{\prime} G}^{\mathbf{i}}}{\epsilon_{k}-E_{f}} f_{\mathbf{i} E \nu}^{\dagger} f_{\mathbf{i} G \nu^{\prime}} c_{k^{\prime} G \nu^{\prime}}^{+} c_{k G \nu^{\prime \prime}}+\frac{V_{k G}^{\mathbf{i} *} \bar{V}_{k^{\prime} E}^{\mathbf{i} G}}{\epsilon_{k}-E_{f}} f_{\mathbf{i} G \nu}^{\dagger} f_{\mathbf{i} G \nu^{\prime}} c_{k^{\prime} E \nu^{\prime \prime}}^{+} c_{k G \nu}+H . c .\right]
$$

These terms being combined with CC exchange term (7) gives additional contributions to the intersite effective exchange, and the first order terms in $\bar{V}$ have the following form,

$$
\bar{H}_{R K K Y}^{n d}=\sum_{\mathbf{i i}^{\prime}} \sum_{E \nu \nu^{\prime} \nu^{\prime \prime}}\left[\bar{I}_{\mathbf{i i}^{\prime}}^{G E} f_{\mathbf{i} G \nu^{\prime}}^{\dagger} f_{\mathbf{i} E \nu} f_{\mathbf{i}^{\prime} G \nu}^{\dagger} f_{\mathbf{i}^{\prime} G \nu^{\prime \prime}}+H . c\right] \text {. }
$$

Here $\bar{I}_{\mathbf{i i}^{\prime}}^{G E}$ is the integral of the same type as that in eq. (13), but the corresponding coupling constant $\alpha^{\prime}$ is proportional to $V^{3} \bar{V}$ and the factors $\bar{B}_{G E}$ and $\bar{K}(T)$ differ from those in (13).

Then, introducing the correlator $\Delta^{G}$ (15), we obtain the mean-field Hamiltonian for the spin-fermion spectrum 円:

$$
H_{M F}=\widetilde{E}_{G} \sum_{\mathbf{i}, \nu} f_{\mathbf{i} G \nu}^{\dagger} f_{\mathbf{i} G \nu}+\sum_{\mathbf{i i}^{\prime}}^{\mathbf{i} \neq \mathbf{i}^{\prime}} \sum_{\nu} \mathcal{T}_{\mathbf{i i}^{\prime}} f_{\mathbf{i}_{G \nu}}^{\dagger} f_{\mathbf{i}^{\prime} G \nu}+\delta H_{M F},
$$

where

$$
\delta H_{M F}=\widetilde{\Delta}_{C F} \sum_{\mathbf{i} \nu} f_{\mathbf{i} E \nu}^{\dagger} f_{\mathbf{i} E \nu}+\sum_{\mathbf{i} \nu \nu^{\prime}}\left[\mathcal{B}_{\mathbf{i i}}^{G E} f_{\mathbf{i} G \nu}^{\dagger} f_{\mathbf{i} E \nu^{\prime}}+H . c .\right]+\sum_{\mathbf{i} \mathbf{i}^{\prime}}^{\mathbf{i} \neq \mathbf{i}^{\prime}} \sum_{\nu \nu^{\prime}}\left[\mathcal{B}_{\mathbf{i} i^{\prime}}^{G E} f_{\mathbf{i} G \nu}^{\dagger} f_{\mathbf{i}^{\prime} E \nu^{\prime}}+H . c .\right]
$$

We use the bare ground state $\mathrm{CF}$ level as a reference point, $E_{G}=0$, then

$$
\widetilde{E}_{G}=-N^{-1} \sum_{\mathbf{k}, k>k_{F}} J_{\mathbf{i}}^{G G}(\mathbf{k}, \mathbf{k})+\sum_{\mathbf{i}^{\prime}}^{\mathbf{i}^{\prime} \neq \mathbf{i}} I_{\mathbf{i i}^{\prime}}^{G G} \equiv B_{c}^{G}+B_{e x}^{G}
$$

includes the covalent and indirect exchange contribution to CF shift determined by the interactions (8) and (11), respectively, and

$$
\widetilde{\Delta}_{C F}=\Delta_{C F}-N^{-1} \sum_{\mathbf{k}, k>k_{F}} J_{\mathbf{i}}^{E E}(\mathbf{k}, \mathbf{k}) \equiv B_{c}^{E}
$$

also includes the one-site covalent corrections. The coupling constant in the MF Hamiltonian is given by the following equation,

$$
\mathcal{T}_{\mathbf{i i}^{\prime}}=\sum_{\nu} I_{\mathbf{i i}^{\prime}}^{G G}\left\langle f_{\mathbf{i}^{\prime} G \nu}^{\dagger} f_{\mathbf{i}^{\prime} G \nu}\right\rangle=I_{\mathbf{i i}^{\prime}}^{G G} \Delta^{G}
$$

Only the sites connected by antiferromagnetic RKKY coupling contribute to the RVB correlation function.

The one-site and intersite mixing constants are determined as

$$
\mathcal{B}_{\mathbf{i i}}^{G E}=-\sum_{\mathbf{k}} \frac{\bar{V}_{k G}^{\mathbf{i} E *} V_{k G}^{\mathbf{i}}}{\epsilon_{k}-E_{f}}
$$

\footnotetext{
${ }^{1}$ The non-diagonal term $\sqrt{12}$ gives no contribution to the uniform mean-field RVB pairing and describes only the fluctuation corrections to the mean-field solutions. These corrections are beyond the framework of the present paper
} 
and

$$
\mathcal{B}_{\mathbf{i i}^{\prime}}^{G E}=\bar{I}_{\mathbf{i i}^{\prime}}^{G E} \Delta^{G}
$$

respectively.

Then, one should diagonalize the quadratic form (19), (20) and obtain the spinon dispersion law $\varepsilon_{\beta}(\mathbf{k})$ ( $\beta$ are the indices of spinon band) under the global constraint condition

$$
(2 N)^{-1} \sum_{\mathbf{k}, \beta}\left(1-\tanh \frac{\varepsilon_{\beta}(\mathbf{k})-\mu}{2 T}\right)=1
$$

which is used in the mean-field approximation instead of exact local constraint (16). This equation determines the spinon chemical potential $\mu$.

Thus, to find the spin-fermion spectrum in the mean-field approximation we need only to diagonalize the Hamiltonian (19), (20) under the constraint (26).

\section{Spinon spectrum of CeNiSn}

CeNiSn crystallizes in the orthorhombic lattice which belongs to the noncentrosymmetric space group $P n 2_{1} a$ [17]. This structure can be described as zigzag chains of Ce atoms directed along $a$ axis (easy magnetization axis) and surrounded by slightly distorted trigonal "drums" formed by $\mathrm{Ni}$ and $\mathrm{Sn}$ ions. Thus, the point symmetry of the crystal field on Ce ions can be treated as nearly trigonal $\left(D_{3 d}\right)$ with rotation axis parallel to a-axis of the crystal, and the monoclinic distortion $\left(C_{s}\right)$ can be considered as a small correction to the trigonal crystal field 《4. Each elementary cell contains four Ce ions.

To apply the theory of spin liquid state explicated in Section 2 to the case of CeNiSn one may use the irreducible representation of the trigonal point group $D_{3 d}$ as a basis for Cornut-Coqblin model and then treat the $C_{s}$ distortion as a perturbation intermixing the trigonal CF terms. It was proposed in [2] (see also [11]) and then confirmed in indirect experiment [4] mentioned in the Introduction, that the ground state level and the first excited level form a pair of Kramers doublets

$$
\begin{aligned}
|G \pm\rangle & =a| \pm 1 / 2\rangle \pm b|\mp 5 / 2\rangle \\
|E \pm\rangle & =| \pm 3 / 2\rangle
\end{aligned}
$$

separated by small energy interval which was estimated as $\approx 4.4 \mathrm{meV}$ in a point charge approximation for the crystal field. Indeed, the purely electrostatic approximation is crude enough, because the covalent and exchange corrections should be taken into account as it is seen from our equations (21) and (22). These corrections result in additional reduction of the CF splitting (see below), so one can be sure that the third CF level is high enough in energy $(\approx 14.0 \mathrm{meV}$

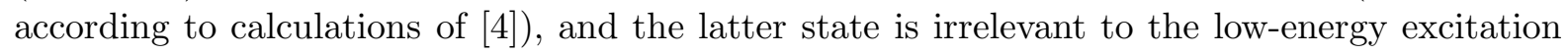
spectrum.

To calculate the spectrum of RVB excitations one should diagonalize the mean field Hamiltonian $H_{M F}+\delta H_{M F}$ given by equations (19) and (20) by means of Fourier transformation in Ce-sublattice which, in turn, has four sublattices in this orthorhombic structure with the use of the basis functions (27,28). This is a cumbersome procedure in general case, but in the special case of CeNiSn one should take into account extreme anisotropy of magnetic response which is seen both in static magnetic susceptibility [3] and in quasi 2D character of neutron scattering 
spectra [7], 8]. These experimental facts enabled us to presume that the magnetic anisotropy reflects the quasi 2D character of low-energy spin excitations. and, hence, to consider the physical situation when these excitations possess the dispersion only in $b c$-plane. The $2 \mathrm{D}$ character of spin-fermion excitations can be explained by the properties of indirect RKKY interaction which is responsible for spinon coupling. It is obvious that only those spins can be involved in the resonating valence bond which are connected by the antiferromagnetic exchange coupling. If one adopts the positive sign of the integral $I_{\mathbf{i}, \mathbf{i}+\rho}^{G G}$ in $b c$ plane ( $\rho$ is the distance between nn Ce ions), one can easily imagine that $15 \%$ lesser value of intersite distance in $a$ direction [17] is enough to have nearly zero or even negative value of oscillating function $\Phi\left(\mathbf{k}_{F}, \mathbf{R}_{i}-\mathbf{R}_{i+\rho}\right)$ in the integral (13). One should emphasize that this assumption does not imply two-dimensionality of conduction electron spectrum which can hardly be imagined in CeNiSn lattice.

Another possible source of anisotropy of magnetic excitation spectrum is the anisotropy of indirect exchange described by the factor $B_{G}(\theta, \phi)$ in eq. (13). The degree of anisotropy given by this mechanism can be estimated for a special case of the ground state (27) (see Appendix). This estimation shows that the "in plane" interaction exceeds the interattion along the $a$-axis when $|a| \gg|b|$ in the case of small interionic distances and when $|a| \ll|b|$ in the case of great interionic spaces. Since the interionic distance in CeNiSn is neither big nor small and the experimental value of $a$ can be estimated as $a \approx 0.67$ (see below), we have no real grounds for referring to this mechanism.

One more reason for changing the sign of $\Phi$ is the anisotropy of the electron Fermi surface. However, the available information about the latter is rather scanty, so we have no firm basis for discussing this mechanism.

In any case the assumption of two-dimensionality is not crucial for the results obtained, as soon as the exchange interaction along $a$-direction is not too big. The brief analysis of the influence of $a$-component of spinon dispersion on the neutron scattering spectra is presented in Subsection 5.4.

Thus, assuming the 2D dispersion of spin liquid excitations, we project the Ce-sublattice onto $b c$ plane and find that the orthorhombic 2D elementary cell contains two Ce ions in the sites $\mathbf{i}=\mathbf{l} \xi$ where $\xi=1,2$ defines the sublattice (see figure 1 ). This network is defined by the Bravais vectors $\mathbf{B}=(b, 0)$ and $\mathbf{C}=(0, c)$ and the basis vector $\mathbf{d}=(0,-b / 2, c / 2-\mathcal{O})$. Here $\mathcal{O}$ is the orthorhombic distortion which transforms one-ion hexagonal lattice into two-ion orthorhombic one.

To describe the 2D spinon spectrum in a nearest neighbour approximation we define the coupling constants $\mathcal{T}_{\mathbf{i i}^{\prime}}$ in equation (23) by two parameters,

$$
\mathcal{T}_{1}=\mathcal{T}_{11, \mathbf{l}^{\prime} 1}=\mathcal{T}_{12, \mathbf{l}^{\prime} 2}
$$

for coupling within the same sublattice, and

$$
\mathcal{T}_{2}=\mathcal{T}_{11,1^{\prime} 2}=\mathcal{T}_{12,1^{\prime} 1}
$$

for intersublattice coupling. Similarly, two mixing integrals $\mathcal{B}_{\mathbf{i i}^{\prime}}$ in equation (24) are introduced, i.e. the one-cite coupling constant 2

$$
\mathcal{G}_{1}=\mathcal{B}_{\mathrm{l} 1,11}^{G E}=\mathcal{B}_{\mathrm{l} 2,12}^{G E}
$$

\footnotetext{
${ }^{2}$ This constant includes also the contribution of monoclinic distortion of electrostatic crystal field
} 
and inter-site coupling constant

$$
\mathcal{G}_{2}=\mathcal{B}_{11,1^{\prime} 2}^{G E}=\mathcal{B}_{12,1^{\prime} 1}^{G E}
$$

Such choice implies that the very existence of two sublattices is due to orthorhombic distortion $\mathcal{O}$ of trigonal lattice, so displacement of second sublattice (see figure 1) is, probably, the main source of the non-Coqblin hybridization. Then, introducing the Fourier transformation of spinon operator,

$$
f_{\mathbf{k} \mu}=N^{-1 / 2} \sum_{\mathbf{l}} \sum_{\xi=1}^{2} \sum_{\Gamma}^{G, E} \sum_{\nu}^{ \pm} \Xi_{\mu}^{\Gamma, \nu}(\xi, \mathbf{k}) f_{\mathbf{l} \xi, \Gamma \nu} \exp (i \mathbf{k} \mathbf{l})
$$

and taking into account the fact that this type of displacement generates the crystal field which components are given by the Stevens operators $\hat{O}_{n}^{2}$ (see also Subsection 5.2), we find the system of equations for the coefficients $\Xi_{\mu}^{\Gamma, \nu}(\xi, \mathbf{k})$ and eigenvalues $\varepsilon_{\mu}(\mathbf{k})$,

$$
\sum_{\mathbf{l}^{\prime}}^{N} \sum_{\xi^{\prime}=1}^{2} \sum_{\Gamma^{\prime}}^{G, E} \sum_{\nu^{\prime}}^{ \pm} \mathcal{D}_{\Gamma \Gamma^{\prime} ; \nu \nu^{\prime}}^{\xi \xi^{\prime}}\left(\mathbf{R}_{l l^{\prime}}\right) \Xi_{\mu}^{\Gamma^{\prime}, \nu^{\prime}}\left(\xi^{\prime}, \mathbf{k}\right) \exp \left\{i \mathbf{k l}^{\prime}\right\}=\varepsilon_{\mu}(\mathbf{k}) \Xi_{\mu}^{\Gamma, \nu}(\xi, \mathbf{k}) \exp \{i \mathbf{k} \mathbf{l}\}
$$

Here the coefficients $D_{\Gamma \Gamma^{\prime}, \nu \nu^{\prime}}^{\xi \xi^{\prime}}\left(\mathbf{R}_{l l^{\prime}}\right)$ are defined as

$$
\begin{array}{r}
\mathcal{D}_{E E ; \nu \nu}^{\xi \xi}(\mathbf{R}=0)=\widetilde{\Delta}_{C F}, \quad \mathcal{D}_{G G ; \nu \nu}^{11}\left(\mathbf{R}=\mathbf{R}_{1}\right)=\mathcal{T}_{1}, \quad \mathcal{D}_{G G ; \nu \nu}^{12}\left(\mathbf{R}=\mathbf{R}_{1}\right)=\mathcal{T}_{2}, \\
\mathcal{D}_{E G ; \nu \nu^{\prime}}^{11}(\mathbf{R}=0)=\mathcal{G}_{1} \quad \mathcal{D}_{E G ; \nu \nu^{\prime}}^{12}\left(\mathbf{R}=\mathbf{R}_{1}\right)=\mathcal{G}_{2}
\end{array}
$$

[see equations (29) - (32)], $\mathbf{R}_{1}$ is the coordinate of the nearest neighboring site. Then the secular matrix can be decoupled into two blocks, $\{(1 G+),(2 G+),(1 E-),(2 E-)\}$ and $\{(1 G-),(2 G-),(1 E+),(2 E+)\}$ which correspond to two new Kramers doublets given by the following linear combinations,

$$
f_{\mathbf{k} \mu, \pm}=\Xi_{\mu}^{G}(1, \mathbf{k}) f_{\mathbf{k} 1, G \pm}+\Xi_{\mu}^{G}(2, \mathbf{k}) f_{\mathbf{k} 2, G \pm}+\Xi_{\mu}^{E}(1, \mathbf{k}) f_{\mathbf{k} 1, E \mp}+\Xi_{\mu}^{E}(2, \mathbf{k}) f_{\mathbf{k} 2, \mp} .
$$

Each block of the secular matrix has the form

$$
\left(\begin{array}{cccc}
\mathcal{T}_{1} M_{1}(\mathbf{k}) & \mathcal{T}_{2} M_{2}(\mathbf{k}) & \mathcal{G}_{1} & \mathcal{G}_{2} M_{2}(\mathbf{k}) \\
\mathcal{T}_{2} M_{2}^{*}(\mathbf{k}) & \mathcal{T}_{1} M_{1}(\mathbf{k}) & \mathcal{G}_{2} M_{2}^{*}(\mathbf{k}) & \mathcal{G}_{1} \\
\mathcal{G}_{1} & \mathcal{G}_{2} M_{2}(\mathbf{k}) & \widetilde{\Delta}_{C F} & 0 \\
\mathcal{G}_{2} M_{2}^{*}(\mathbf{k}) & \mathcal{G}_{1} & 0 & \widetilde{\Delta}_{C F}
\end{array}\right)
$$

The structure factors which define the spinon dispersion are determined as

$$
\begin{gathered}
M_{1}(\mathbf{k})=2 \cos (\mathbf{k B}) \\
M_{2}(\mathbf{k})=4 \exp \left(i \frac{\mathbf{k B}-\mathbf{k} \mathbf{C}}{2}\right) \cos \left(\frac{\mathbf{k B}}{2}\right) \cos \left(\frac{\mathbf{k C}}{2}\right)
\end{gathered}
$$

$(\mathbf{B}=(b, 0)$ and $\mathbf{C}=(0, c))$.

The excitation spectrum is shown in figure 2 (the insert presents the dispersion of spin excitations in Coqblin-Cornut approximation $\left(\mathcal{G}_{1}=0, \mathcal{G}_{2}=0\right.$ ) where the CF excitations are simply superimposed on the ground state spinon band). It is seen from this figure that the intermixing of spinon and $\mathrm{CF}$ excitations results in radical reconstruction of the low-energy part of this spectrum. We associate the characteristic temperature $T^{*}$ estimated from various experiments 
as $T^{*} \approx 80 \mathrm{~K}$ (see Introduction) with the characteristic energy scale of this structured part, so the values of $\mathcal{T}_{1}=12.7 \mathrm{~K}$ and $\mathcal{T}_{2}=18 \mathrm{~K}$ were chosen to reproduce approximately the width of this energy interval. The values of other parameters $\left(\mathcal{G}_{1}, \mathcal{G}_{2}\right.$ and $\left.\widetilde{\Delta}_{C F}\right)$ where taken to fit the positions of the main peaks in the neutron scattering spectra (see the next section). In particular, we adopted the value of $13.5 \mathrm{~K}$ for the renormalized $\mathrm{CF}$ splitting parameter $\widetilde{\Delta}_{C F}$ ). Its reduction in comparison with the value of $48.4 \mathrm{~K}$ given by indirect experiment of [4] is easily explained by the effects of covalent and exchange renormalization given by the terms $B_{c}^{G}, B_{c}^{E}$ and $B_{e x}^{G}$ in eqs. (21) and (22). It is obvious that the covalent repulsion $B_{c}^{\Gamma}$ of the localized levels $E_{\Gamma}$ from the free conduction band states above the Fermi level (21,22) is governed by the value of hybridization matrix elements $\left|V_{k \Gamma}^{\mathbf{i}}\right|^{2}$. Since the Fermi surface of CeNiSn is dominated by the $p$-partial waves [19], the hybridization is largest for the $j_{z}= \pm 1 / 2$ and $j_{z}= \pm 3 / 2$ components of the f-states. Therefore, the ratio of hybridization matrix elements for $|G \pm\rangle$ (27) and $|E \pm\rangle$ (28) can be evaluated as $\left|V_{k G \pm}^{\mathbf{i}}\right|^{2} /\left|V_{k E \pm}^{\mathbf{i}}\right|^{2} \approx a^{2}<1$. This means that the negative covalent term reduces the $\mathrm{CF}$ splitting. The exchange contribution $B_{e x}^{G}$ in (21) gives additional contribution to this reduction effect.

The dispersionless behavior of spinon spectrum at the $b$-facet of Brillouin zone $[(0,1 / 2,0) \leq$ $\mathbf{k} \leq(0,1 / 2,1 / 2)]$ is explained by the specific form of the spinon spectrum

$$
\varepsilon_{\mu=1,2}(\mathbf{k})=2\left\{\mathcal{T}_{1} \cos (\mathbf{k B}) \pm 2 \mathcal{T}_{2} \cos \left(\frac{\mathbf{k B}}{2}\right) \cos \left(\frac{\mathbf{k} \mathbf{C}}{2}\right)\right\}
$$

since this spectrum becomes dispersionless for $\mathbf{k B}=\pi$.

It is important that inclusion of intermixing terms $\mathcal{G}_{1,2}$ in the secular matrix (37) does not alter the one-dimensionality of this branch (cf. upper and lower panels of figure 2) because the form factor $M_{2}$ given by eq. (39) also turns into zero at $\mathbf{k B}=\pi$. Basing on the spinon dispersion law shown in this picture we will try in the next section to explain the neutron scattering data. The chemical potential of spin-fermions at $T=0$ which is determined by the constraint (26) is shown in this picture by the dashed line.

Thus we find that the spin excitations in CeNiSn preserve to some extent the features of the one-site crystal field excitation spectrum: the multiband dispersion picture contains vast dispersionless parts which origin is the initial atomic crystal field splitting of the f-level. The density of spin-fermion states described by these bands is shown in upper and lower panel of figure 3. We see that instead of simple two-level picture of CF excitations there exists several peaks some of which can be considered as the remnants of CF levels but other reflect the van Hove singularities of $2 \mathrm{D}$ spinon spectrum. The spectrum demonstrates the pseudogap features presupposed in early phenomenological descriptions [3]. However, it is remarkable that the chemical potential of spin-fermion excitations falls not into the hybridization gap but into the pseudogap between two van Hove peaks.

\section{Inelastic Neutron Scattering in Spin Liquid}

The inelastic neutron scattering provides unique experimental information concerning the structure and dispersion of low-energy spin excitations, so these data look challenging for the general theory of spin liquid and especially for the case of interplay between $\mathrm{HF}$ anf $\mathrm{CF}$ excitations. To

calculate the spectra of inelastic neutron scattering in the spin liquid, we start with the well 
known equation for magnetic scattering cross section

$$
\frac{d^{2} \sigma}{d \Omega d E^{\prime}}=\left(\frac{\gamma e^{2}}{m_{e} c^{2}}\right)^{2} \frac{\kappa^{\prime}}{\kappa} \sum_{\alpha \beta}\left(\delta_{\alpha \beta}-\hat{Q}_{\alpha} \hat{Q}_{\beta}\right) S_{\alpha \beta}(\mathbf{Q}, \hbar \omega)
$$

Here $m_{e}$ and $e$ are the mass and the charge of electron, $\gamma=-1.91$ is the gyromagnetic ratio for neutron, $c$ is the light velocity. The wave vectors $\kappa$ and $\kappa^{\prime}$ are momenta of incident and scattered neutron, respectively, and $\hat{Q}_{\alpha}$ are the cartesian components of unit vector directed along the momentum transfer vector $\mathbf{Q}$. The component $S_{\alpha \beta}(\mathbf{Q}, \hbar \omega)$ of the scatering function can be expressed as the sum over all possible initial $(\lambda)$ and final $\left(\lambda^{\prime}\right)$ states with the energies $E_{\lambda}$ and $E_{\lambda}$, respectively,

$$
S_{\alpha \beta}(\mathbf{Q}, \hbar \omega)=\sum_{\lambda \lambda^{\prime}} n_{\lambda}\left\langle\lambda\left|\hat{\mathcal{Q}}_{\alpha}^{+}\right| \lambda^{\prime}\right\rangle\left\langle\lambda^{\prime}\left|\hat{\mathcal{Q}}_{\beta}\right| \lambda\right\rangle \delta\left(\hbar \omega+E_{\lambda}-E_{\lambda^{\prime}}\right)
$$

( $n_{\lambda}$ is the probability distribution for initial state). Then, the operator $\hat{\mathcal{Q}}$ of neutron-electron interaction can be expressed as a sum over all $\mathrm{f}$ ions,

$$
\hat{\mathcal{Q}}=\sum_{\mathbf{l}} \exp \left(i \mathbf{Q} \mathbf{R}_{l}\right) \sum_{\xi}^{\text {cell }} \exp \left(i \mathbf{Q} \mathbf{d}_{\xi}\right) \hat{\mathcal{Q}}_{l \xi}
$$

In the dipolar approximation the operator $\hat{\mathcal{Q}}_{l \xi}$ turns into

$$
\hat{\mathcal{Q}}_{l \xi}=\frac{1}{2} g F(\mathbf{Q}) \hat{\mathbf{J}}_{l \xi}
$$

where $\mathrm{g}$ is the Lande splitting factor, $F(\mathbf{Q})$ is the ionic fomfactor and $\hat{\mathbf{J}}$ is the total angular momentum operator. Thus the problem is reduced to calculation of the correlator $\left\langle\lambda\left|\hat{J}_{l \xi}^{+} \hat{J}_{l^{\prime} \xi^{\prime}}^{-}\right| \lambda\right\rangle$

In our model the source of neutron scattering are the transitions between the spin liquid excitations described by the operators (33) with the dispersion law $\varepsilon_{\mu}(\mathbf{k})$ given by the secular equation (37). Now the situation formally resembles the well-known paramagnetic scattering [18] where the spin-fermion excitations $\lambda=\mathbf{k} \mu$ play the same part as the itinerant electrons in transition metals. Substituting the eigen vectors given by equation (33) in the scattering function (42), we find

$$
\begin{gathered}
S_{\alpha \beta}(\mathbf{Q}, \hbar \omega)=\left(\frac{1}{2} g F(\mathbf{Q})\right)^{2} \\
\sum_{\mu \mu^{\prime}} \sum_{\mathbf{k}}\left[I_{\alpha}^{\mu \mu^{\prime}}(\mathbf{k}, \mathbf{Q})\right]^{*} I_{\beta}^{\mu \mu^{\prime}}(\mathbf{k}, \mathbf{Q}) n_{\mu}(\mathbf{k})\left[1-n_{\mu^{\prime}}(\mathbf{k}-\mathbf{Q})\right] \delta\left(\hbar \omega+\varepsilon_{\mu}(\mathbf{k})-\varepsilon_{\mu}(\mathbf{k}-\mathbf{Q})\right)
\end{gathered}
$$

where

$$
I_{\alpha}^{\mu \mu^{\prime}}(\mathbf{k}, \mathbf{Q})=\sum_{\Gamma \nu} \sum_{\Gamma^{\prime} \nu^{\prime}} \sum_{\xi} \exp \left(i \mathbf{Q} \mathbf{d}_{\xi}\right)\left(\Xi_{\mu}^{\Gamma, \nu}(\xi, \mathbf{k})\right)^{*} \Xi_{\mu^{\prime}}^{\Gamma^{\prime}, \nu^{\prime}}(\xi, \mathbf{k}-\mathbf{Q})\left\langle\Gamma \nu\left|\hat{J}^{\alpha}\right| \Gamma^{\prime} \nu^{\prime}\right\rangle
$$

$n_{\mu}(\mathbf{k})$ is the Fermi distribution function for spinon excitations

This expression can be simplified in case of CeNiSn. It is known from experiment [9, 10] that the dominant component which contributes significantly to the scattering function is $S_{a a}(\mathbf{Q}, \hbar \omega)$ 
component. Thus, for the momentum transfer $\mathbf{Q}$ in the $b-c$ plain, which is the experimental condition for the most of experimental scans, one has for the scattering function

$$
\begin{gathered}
S_{a a}(\mathbf{Q}, \hbar \omega)=\left(\frac{1}{2} g F(\mathbf{Q})\right)^{2} \\
\sum_{\mu \mu^{\prime}} \sum_{\mathbf{k}}\left|I_{a}^{\mu \mu^{\prime}}(\mathbf{k}, \mathbf{Q})\right|^{2} n_{\mu}(\mathbf{k})\left[1-n_{\mu^{\prime}}(\mathbf{k}-\mathbf{Q})\right] \delta\left(\hbar \omega+\varepsilon_{\mu}(\mathbf{k})-\varepsilon_{\mu}(\mathbf{k}-\mathbf{Q})\right)
\end{gathered}
$$

Since the axis $a$ is the axis of spin quantatisation, and since the operator $J_{a}$ has only diagonal nonzero matrix elements in the basis of CF states, $|\Gamma \nu\rangle(27,28)$, the expression for $I_{\alpha}^{\mu \mu^{\prime}}(\mathbf{k}, \mathbf{Q})$ has the form

$$
I_{a}^{\mu \mu^{\prime}}(\mathbf{k}, \mathbf{Q})=\sum_{\Gamma \nu} \sum_{\xi} \exp \left(i \mathbf{Q} \mathbf{d}_{\xi}\right)\left(\Xi_{\mu}^{\Gamma, \nu}(\xi, \mathbf{k})\right)^{*} \Xi_{\mu^{\prime}}^{\Gamma, \nu}(\xi, \mathbf{k}-\mathbf{Q})\left\langle\Gamma \nu\left|\hat{J}^{a}\right| \Gamma \nu\right\rangle
$$

Finally, taking into account the explicit equation (36) for the spinon operator and expressions for the CF states (27,28) one comes to the final equation for the matrix element $I_{\alpha \pm}^{\mu \mu^{\prime}}(\mathbf{k}, \mathbf{Q})$ (signs \pm are for up and down partner of the Kramers doublet (36) respectively)

$$
\begin{aligned}
I_{\alpha \pm}^{\mu \mu^{\prime}}(\mathbf{k}, \mathbf{Q})= & {\left[\left(\Xi_{\mu}^{G}(1, \mathbf{k})\right)^{*} \Xi_{\mu^{\prime}}^{G}(1, \mathbf{k}-\mathbf{Q}) \Theta_{G \pm}+\left(\Xi_{\mu}^{E}(1, \mathbf{k})\right)^{*} \Xi_{\mu^{\prime}}^{E}(1, \mathbf{k}-\mathbf{Q}) \Theta_{E \mp}\right]+} \\
& {\left[\left(\Xi_{\mu}^{G}(2, \mathbf{k})\right)^{*} \Xi_{\mu^{\prime}}^{G}(2, \mathbf{k}-\mathbf{Q}) \Theta_{G \pm}+\left(\Xi_{\mu}^{E}(2, \mathbf{k})\right)^{*} \Xi_{\mu^{\prime}}^{E}(2, \mathbf{k}-\mathbf{Q}) \Theta_{E \mp}\right] } \\
& \times \exp (i \mathbf{Q d})
\end{aligned}
$$

where

$$
\begin{gathered}
\Theta_{G \pm}= \pm\left(3 a^{2}-\frac{5}{2}\right) \\
\Theta_{E \pm}= \pm \frac{3}{2}
\end{gathered}
$$

\section{Inelastic neutron scattering spectra in CeNiSn}

In this section we compare the inelastic neutron scattering spectra calculated by means of eqs. (47) and (49) with dispersion law for spin-fermions given by secular matrix (37) and figure 2 with the experimental data of the refs. [7, 8, 9, 10]

\subsection{Qualitative considerations}

The inelastic neutron scattering spectrum of CeNiSn in its gross features is formed by two main signals with the energies of $\sim 2 \mathrm{meV}$ and $\sim 4 \mathrm{meV}$. It is seen from the lower panels of figures 2 and 3 that these "peaks" can be ascribed to transitions from the occupied states in the lower band to the region of the empty levels where the states $|G \pm\rangle(27)$ and $|E \pm\rangle(28)$ are strongly hybridized. The most distinctly these features are seen in experiment for the momentum transfer vector

$\mathbf{Q}$ in the $b c$-plain and, hence, the magnetic response is connected with the $\chi^{a a}$ component of dynamic magnetic susceptibility [9]. As is known from the measurements of static succeptibility, the $a$-axis is the easy magnetization axis in CeNiSn [3]. Since the lower spinon band is formed mainly by the ground state doublet $|G \pm\rangle$ (27) where the components $| \pm 5 / 2\rangle$ play the main 
part (see next subsection), the longitudinal susceptibility should be the strongest component of magnetic response at low energies, so the dominance of $\mathbf{Q} \perp a$ momentum transfer vectors is naturally explained in our model. Then the most intense transitions ar expected for those final states where the greater fraction of $| \pm 5 / 2\rangle$ is admixed to the $| \pm 3 / 2\rangle$ states.

The $2 \mathrm{meV}$ peaks are seen experimentally for $\mathbf{Q}=(0,0,1)$ [7] and $\mathbf{Q}=(0,1,0)$ [8, 9]. The corresponding momentum transfer vectors are indicated by solid arrows in figure 4 where the shaded area is the projection of Brillouin zone to the $b c$ plane). These processes correspond to the vertical transitions from the spinon "Fermi surface" to the region of strong hybridization near the point $\mathbf{k}=(0,0,1 / 2)$. The bold arrow marks these transitions in figure 2 (the integer reciprocal vector $(0,1,0)$ or $(0,0,1)$ are substracted). The same transitions are shown by the bold arrow in the lower panel of figure 3. The highest peak in DOS arises due to the van Hove singularities near the former $\mathrm{CF}$ excitation level.

The peaks at $E=4 \mathrm{meV}$ are seen for $\mathbf{Q}=\left(0,1 / 2+n, Q_{c}\right)$ (grey arrows in figure 4) where $n$ are integer numbers [8, 9]. This maximum in the scattering cross section is due to the intraband transitions shown by grey arrows in figure 2 . It is easily seen that since the $4 \mathrm{meV}$ peak corresponds to the transitions between two peaks of DOS (grey arrow in the lower panel of figure 3 ), and the $2 \mathrm{meV}$ processes are due to the transitions from the structurless part of DOS to the empty peak, the intensity of the former signal should be higher than that of the latter one. This result is also consistent with the experimental data [8, 9].

It worth to be noted that the general shape of the DOS in the lower band reminds the doublepeak DOS with pseudogap postulated in early phenomenological models [3, 2, 6]. However, at least one more peak with higher energy should exist in the scattering spectrum given by the present model. This peak is formed by the interband transitions shown by the dashed lines in figures 2 and 3 . Such peak with $E \approx 7 \mathrm{meV}$ was found in the recent neutron scattering experiments 10].

Thus, the present model explains all qualitative features of the neutron scattering spectrum. To make the detailed quantitative description of experimental scans one should first specify the model parameters.

\subsection{Evaluation of the model parameters}

To calculate the spinon spectrum determined by the secular matrix (37) one should know the values of the intersite coupling constant $\mathcal{T}_{1,2}$, the mixing parameters $\mathcal{G}_{1,2}$ the energy $\widetilde{\Delta}_{C F}$ of $\mathrm{CF}$ splitting. To find the intensities of neutron scattering one needs also the magnitude of the orthorhombic distortion $\mathcal{O}$ and the values of parameters $a, b$ which determine the structure of the ground state $\mathrm{CF}$ level $|G \pm\rangle$ (27). All these quantities, except hybridization parameters $\mathcal{G}_{b c}$ and $\mathcal{G}_{0}$ are, indeed, predetermined by the experimental data available. The criterions for choosing the parameters $\mathcal{T}_{1,2}$ and $\widetilde{\Delta}_{C F}$ are described in Section 3. The value of orthorhombic distortion $\mathcal{O}$ in units of lattice parameter $c$ is 0.10 [17.

We have no independent experimental information to choose the values of mixing parameters $\mathcal{G}_{1,2}$, so we use these parameters for fitting the experimental neutron scattering cross section. The best values, which gave us the possibility to describe the low-energy magnetic scattering function for all measured momentum transfer are $\mathcal{G}_{1}=-3.5 \mathrm{~K}, \mathcal{G}_{2}=-7.7 \mathrm{~K}$.

To estimate the parameters $a, b$ entering the ground state CF level wave function (27) we used the experimental data on the anizotropy of low temperature static magnetic susceptibility at low temperatures [5]. According to our model, this susceptibility should be mainly determined 
by the Pauli-like contribution of RVB excitations.

The Pauli-like contribution for magnetic field applied along the $j$-axis at low temperatures is proportional to the DOS of spinons $N\left(\varepsilon_{F}\right)$ and to the square of effective $g_{j}^{(p)}$-factor of the spinons states $|\widetilde{G} \pm\rangle$ at the Fermi level, $\chi_{x}^{(p)} \sim\left|g_{x}^{(p)}\right|^{2} \mathcal{N}\left(\varepsilon_{F}\right)\left(\mathcal{N}\left(\varepsilon_{F}\right)\right.$ is the density of spinon states at the Fermi level). The effective $g_{j}^{(p)}$-factor is determined as $g_{j}^{(p)}=2 g_{J}\left|\left\langle\widetilde{G} \nu\left|\hat{J_{j}}\right| \widetilde{G} \nu^{\prime}\right\rangle\right|(\nu= \pm)$. Here $g_{J}=6 / 7$ is the Lande factor for $\mathrm{Ce}^{3+}$ and $\hat{J}_{j}$ is the $j$ - component of total momentum operator. According to (36), the spinon states at the Fermi surface which determine the anisotropy of the susceptibility are described as the mixture of the bare wave functions $|G \pm\rangle$ and $|E \pm\rangle(27),(28)$. due to orthorhombic distortion of the trigonal lattice. As was mentioned above, we consider the $\hat{O}_{n}^{2}$ orthorhombic distortion (see Fig. 1). Therefore, the wavefunctions in the vicinity of the Fermi surface can be approximated by

$$
|\widetilde{G} \pm\rangle=\alpha_{a v}^{(F)}|G \pm\rangle+\beta_{a v}^{(F)}|E \mp\rangle
$$

where $\alpha_{a v}^{(F)}$ and $\beta_{a v}^{(F)}$ are the mixing parameters averaged over the Fermi surface.

When evaluating the Pauli contribution, we neglect the anisotropy of spinon Fermi surface and assume that the ratio between the components of static susceptibility is given by the anisotropy of $g$ factor:

$$
\chi^{a}: \chi^{b}: \chi^{c} \approx\left|g_{a}\right|^{2}:\left|g_{b}\right|^{2}:\left|g_{c}\right|^{2}
$$

To evaluate the averaged mixing parameters $\alpha_{a v}^{(F)}$ and $\beta_{a v}^{(F)}$ we take into account that there are $N=4$ nearest neighbors from the second sublattice which take part in k-dependent hybridization. The averaging of the k-dependence of hybridization gives additional factor $1 / 2$. The distance from the Fermi surface $\varepsilon_{F}$ to the crystal field level is $\approx 30 \mathrm{~K}$ according to our calculations (see figures 2,3). Therefore, the value of $\beta_{a v}$ at the Fermi surface can be estimated as $\beta_{a v}^{(F)} \approx N \mathcal{G} / 2\left(\Delta_{C F}-\varepsilon_{F}\right)$, so $\alpha_{a v} \approx 0.88$. The similar value of parameter parameter $\alpha_{a v}$ can be extracted from the experimental data for the anisotropy of magnetic susceptibility

$$
s_{1}=\frac{\left|\chi_{b}-\chi_{c}\right|}{\chi_{c}} \approx\left(\frac{4 \sqrt{2} \sqrt{1-\alpha_{a v}^{2}}}{3 \alpha_{a v} a}\right)^{2}
$$

(where $s_{1} \approx 0.25$ [5]). One finds $\alpha_{a v} \approx 0.94$ from (53) in agreement with the theoretical evaluation. The estimation of the magnetic susceptibility gives

$$
s_{0} \equiv \frac{\chi_{a}}{\chi_{c}} \approx \frac{\chi_{a}}{\chi_{b}} \approx\left(\frac{\alpha_{a v}\left(2-6 a^{2}\right)+3}{3 \alpha_{a v}^{2} a^{2}}\right)^{2}
$$

where $2<s_{0}<3$. Using (54) the value of $|a|$ is determined as

$$
|a| \approx \sqrt{\frac{2 \alpha_{a v}^{2}+3}{3 \alpha_{a v}^{2}\left(2+\sqrt{s_{0}}\right)}}
$$

so we obtain $0.65<|a|<0.75$. Since the contribution of $|5 / 2\rangle$-component in the magnetic response is dominant even for $|a| \approx|b|$, we actually deal with the $| \pm 5 / 2\rangle$ states in the neutron scattering experiments. It turns out that the neutron scattering cross sections weakly depend on the the value of $a$ in the actual range of $|a|$, and we can not extract this parameter from the available experimental data. In the following calculations the value of $|a|=0.67$ is taken. 


\subsection{Quantitative description of constant-Q and constant-E neutron scatter- ing scans}

First, we see that our model reproduces quite well the "pseudogap" behavior of constant-Q scans first found in [7]. Figure 5 presents the theoretical curves for $\mathbf{Q}$ perpendicular to a axis in comparison with experimental data of ref. [7] and later results of [8, [9]. It is easily seen from Figs 2 and 3 (lower panel) that this character of $\mathbf{Q} \perp$ a scans is due to high density of states for the $| \pm 5 / 2\rangle$ components in the region of strong hybridization around $(0,0,1 / 2)$ point. This psedogap-like behavior disappears for $\mathbf{Q} \| \mathbf{a}$ [7] simply because the 2-meV peak is suppressed for this direction of momentum transfer in accordance with the geometrical factor in eq. (41) under condition $\chi_{a a} \gg \chi_{b b}, \chi_{c c}$.

The next striking feature of experimental scans is the quasi $1 \mathrm{D}$ character of $4-\mathrm{meV}$ excitation: it was noticed [8, 9] that the behavior of this peak for $\mathbf{Q}=\left(0, n+1 / 2, Q_{c}\right)$ practically does not depend on the value of $Q_{c}$. Our next scan demonstrates, first, that this peak is indeed can be obtained in the spin-fermion model (figure 6, upper panel) and, second, that nothing is changed significantly in the shape of this scan with varying $Q_{c}$ (lower panel). The form of spinon dispersion curve explains this one-dimensionality: due to the dispersionless character of the energy band along the wave vector $\mathbf{k}$ in $\mathbf{k}=\left(0,1 / 2, k_{c}\right)$ direction [see equation (40) for explanation], the contribution from the intraband transitions with $E \sim 4 \mathrm{meV}$ to the scattering function (spanted arrows in fiture $2 \mathrm{~b}$ ) does not depend on $Q_{c}$ and thus mimics quasi onedimensional scattering.

One more remarkable property of experimental constant- $\mathbf{Q}$ scans is their modification along the $\left(0, Q_{b}, 0\right)$ direction. It was found (see [9], figure 3) that the form of these scans change from the structure with definite $4.2 \mathrm{meV}$ peak at $Q_{b}=1.5$ to that with $2 \mathrm{Mev}$ peak at $Q_{b}=1$. It is seen from figure 7 that our theoretical scans reproduce this variation quite satisfactorily, including the featureless shape of the scan at intermediate value of $Q_{b}=1.2$.

The theory explains also why the $2 \mathrm{meV}$ peak which is distinctly seen for the vertical transitions $\left(0, Q_{b}, 0\right)$ and $\left(0,0, Q_{b}\right)$ (see figures 4,5$)$ practically disappears for the "diagonal" direction $\mathbf{Q}=(0,1,1)$ (figure 16 of [9]). This kind of anisotropy is explained by the fact that the phase factor $\exp (i \mathbf{Q} \mathbf{d})$ in $(49)$ is close to unity for $\mathbf{Q}=(0,1,1)$ due to the small orthorhombic shift $\mathcal{O}=0.10$ (see Subsection 5.2). Then, since the condition $\Theta_{G \pm} \approx \Theta_{E \mp}$ is valid for the chose value of $a=0.67$, the matrix element (48), (49) practically turns out into the orthogonality relation between the different quantum states (33) with the same reduced wave vector. This is why the intensity of scan at $\mathbf{Q}=(0,1,1)$ is by order of magnitude smaller than that of $\mathbf{Q}=(0,0,1)$ or $\mathbf{Q}=(0,1,0)$.

Finally, we discuss the characteristic features of constant-E scans. The calculated scans for $E=4.2 \mathrm{meV}$ are presented in figure 8 together with the experimental data taken from [9]. It is no wonder that the theoretical scans are in satisfactory quantitative agreement with experiment: it simply follows from above explanation that the maxima of $\left(0, Q_{b}, 0\right)$ and $\left(0, Q_{b}, 1\right)$ scans at $Q_{b}=0.5,1.5$ are connected with the fact that the $4 \mathrm{meV}$ peak arises just at $\mathbf{Q}=(0, n+1 / 2, x)$ with integer $n$ and any $x$.

More puzzling feature of the constant-E scans was noticed in [10] where it was observed that the peak at $Q_{b}=1.5$ for the $\left(0, Q_{b}, 1\right)$ scans with $E=4.2 \mathrm{meV}$ splits into two peaks for lower energy transfer $E=3.3 \mathrm{meV}$ and $E=2.5 \mathrm{meV}$. This feature is reproduced, at least quantitatively, in our calculations (see figure 9). Both the appearance of the doubled peak and the increase of the splitting for lower energies is seen in the theoretical curves. Even the higher 
intensity of the left peak is reproduced in the calculated scan. To investigate the reason of this asymmetry we calculated also the constant- $\mathbf{Q}$ scans with $\mathbf{Q}=\left(0, Q_{b}, 1\right)$ for $Q_{b}=1.25,1.51 .75$. Our figure 10 demonstrates that for higher energy transfer the intensity of $Q_{b}=1.5$ response is higher than that of $Q_{b}=1.25,1.75$ whereas for lower energy transfer the situation is reversed.

Thus, the totality of calculated scans shows that not only the general shape but also the anisotropy of spinon spectrum correlates with the details of experimental neutron scattering scans. These results demonstrate clearly that the anisotropic magnetic response of CeNiSn is due to anisotropy of the spectrum of low-energy spin-fermion excitations in this system.

\subsection{Influence of 3-dimensionality of the spinon spectrum}

To check the robustness of our results to assumption about mainly two-dimensional character of spinon dispersion we recalculated the spinon DOS and inelastic neutron scattering intensities.

We introduced in the secular matrix for the spinon spectrum two more parameters, i.e. the "overlap integral" $\mathcal{T}_{3}=\mathcal{T}_{11,1^{\prime} 3} \equiv \mathcal{T}_{12,1^{\prime} 4}$ where the indices $(3,4)$ stand for the Ce ions above and below the sites $(1,2)$ respectively, in a-direction, and $\mathcal{G}_{3}=\mathcal{B}_{11,1^{\prime} 3}^{G E} \equiv \mathcal{B}_{12,1^{\prime} 4}^{G E}$ with the same notations. These parameters result in further doubling of the crystal cell lattice and further removing the degeneracy of spinon spectrum and appearance of spinon dispersion in $a$-direction of the Brillouin zone. As to the spinon DOS, the overlap parameter $\mathcal{T}_{3}$ is responsible mainly for for broadening of the second van Hove peak ( $-25 \mathrm{~K}$ peak in the lower panel of fig.3) and the influence of the hybridization parameter $\mathcal{G}_{3}$ is seen in broadening and lowering of the 3 $\mathrm{K}$ peak in the mentioned DOS. But the general shape of the DOS, and, therefore, the form of the inelastic neutron scattering spectra for $\mathbf{Q}$ in $b c$-plane are practically not modified at least for $\mathcal{T}_{3}$ and $\mathcal{G}_{3}$ less then $\approx 4 K$, i.e. more then 50 per cent of the characteristic values of inplane interaction parameters. Correspondingly, the neutron scattering peaks for the momentum transfer in $b c$ plane are also slightly broadened, but the general quantitative agreement is still satisfactory, although the CF splitting parameter should be slightly changed. If one chooses, e.g., $\mathcal{T}_{3}=\mathcal{G}_{3}=3.5 \mathrm{~K}$, the renormalized value of $\tilde{\Delta}_{C F}=19.5 \mathrm{~K}$ should be taken to get an agreement with the experimental data for all scans demonstrated in figures 5-10.

However, the introduction of third component in spinon spectrum provided us one more possibility to check the model assumptions, i.e. to calculate the neutron scattering for $\mathbf{Q}$ out of $b c$-plane. In fig.11 the constant-E scan for $\mathbf{Q}=\left(Q_{a}, 3 / 2,0\right)$ is presented in comparison with the experimental data of [9]. The oscillating character of scattering intensity with $Q_{a}$ is distinctly seen in experiment and is shurely reproduced in the calculated curve. It is interesting that the shape of the theoretical curve is more complicated than the rough sinusoidal guideline shown in fig.11d of Ref. [9], and the attentive look on the experimental points confirms this picture at least qualitatively.

When calculating the theoretical curve we weighted the inelastic cross section only with the form factor $F(\mathbf{Q})$ [see eq. (45)]. This procedure resulted in better agreement with the

experiment than that including also the orientational factor $1-\hat{Q}_{a}^{2}$. This means that the contribution of $\operatorname{Im} \chi^{b b}$ and $\operatorname{Im} \chi^{c c}$ to this cross section is also significant due to, e.g., the zigzag distortion of Ce chains an $a$-direction. 


\section{Low-temperature specific heat of CeNiSn}

To be sure that our approach gives really universal description of low-energy spin excitations in CeNiSn, we calculated the magnetic contribuion to specific heat of CeNiSn within the approximate mean-field approach to RVB thermodynamics with the use of the same parameters for the spin fermion spectrum which were chosen for description of the neutron scattering data. Since the samlpes studied in the neutron scattering measurements, apparently, were not as perfect as the recently grown samples which demonstrate the metallic type conductivity and low value of $\gamma=C / T \sim 40 \mathrm{~mJ} / \mathrm{mole}^{2}$ [5], we used for comparison the data for the sample \#2 from [5]. The value of $\gamma(T=0.1 K)=80 \mathrm{~mJ} / \mathrm{mole}^{2}$ was chosen to eliminate the ultralow- $\mathrm{T}$ upturn due to nuclear and impurity contribution to the magnetic specific heat. The results of our calculations are presented in fig. 12 (solid curve). The experimental data presented by diamonds in figure 12 are obtained from those for that sample by cancelling the phonon contribution to specific heat. The latter was considered to be the same as in LaNiSn crystal [20].

When comparing the experimental and theoretical curves for the specific heat, one should take into account the contribution of conduction electrons and add it to the magnetic specific heat given by the spin fermion excitations. We have taken it to be linear in $\mathrm{T}$ within the low- $\mathrm{T}$ interval $0<T<10 \mathrm{~K}$ with the Sommerfeld coefficient $\gamma_{e}=18 \mathrm{~mJ} / \mathrm{mol} \mathrm{K}^{2}$. This means that the electron contribution to the heavy fermion density of states in good samples can be as large as $\approx 50$ per cent in agreement with the theoretical predictions for the spin liquid state of the Kondo lattices [22]. It is seen from figure 12, that the theoretical curve describes quite well the temperature behavior of $C / T=\gamma+b T$ at low temperture and gives the maximum of $C / T$ at $T=6 \mathrm{~K}$. The latter is observed for the more perfect samples [21] where the data for higher temperatures are available.

It should be noted that the contribution of spinon dispersion in $a$-direction (see Section 5.4) practically does not change the form of $C(T)$ curve since the van Hove peaks survive in 3D spectrum, and the spinon chemical potential is still in the dip of the DOS.

Our previous semi-phenomenological studies have shown that the model of heavy fermion liquid with the pseudogap in the spin-fermion excitations induced by the interplay with the $\mathrm{CF}$ excitations describes quantitatively the low-temperature specific heat and thermal expansion of CeNiSn [2, 11]. Now we see that the consequent microscopic theory gives the detailed picture of the low-energy excitations and low-temperature thermodynamics of this system which is in reasonable quantitative agreement with the experiment.

\section{Concluding remarks}

To conclude, we have found that the general theory of spin-fermions acquires some specific features in the low-symmetry Kondo lattice materials with soft CF excitations, such as CeNiSn and CeRhSb. These CF excitations modify the spectrum of spin excitations in a very characteristic manner, and as a result, the density of spin-fermion states has several peaks due to interaction between the spin-fermion excitations and CF excitations. Because of existence of these peaks the spin response of CeNiSn and CeRhSb reminds that of spin-gap materials.

Whereas the largest peak in spinon DOS at $\approx 2 \mathrm{meV}$ above the spinon Fermi level appears as a result of interplay between the $\mathrm{HF}$ and $\mathrm{CF}$ excitations, all other singularities in one-spinon and two-spinon DOS arise due to quasi two-dimensionality of spin excitation spectrum (although these singularities are robust enough against the appearance of the third component of spinon 
dispersion). The source of this anisotropy of the excitation spectrum is the anisotropy of RKKY interaction in a real space. It is worth mentioning that the standard slave-boson description used in a previous phenomenological model [2, 11] also predicts highly anisotropic spectrum of HF excitations due to the sf-hybridization anisotropy in $\mathbf{k}$-space.

If the levels $|\mp 5 / 2\rangle$ dominate in the ground state Kramers doublet $|G \pm\rangle$ (27) (see Section 5.2), then the effective intersite overlap integral $\mathcal{T}_{\mathbf{i j}}^{G G}$ is determined in this theory by the combination of hybridization matrix elements, $V_{\mathbf{k} \Lambda}^{\mathbf{i} *} V_{\mathbf{k} \Lambda^{\prime}}^{\mathbf{j}} /\left(\epsilon_{F}-E_{f}\right)$, which turns into zero for $\mathbf{k}=$ [1.0.0] (see, e.g., [23]). This means that the f-electron wave functions form the non-bonding states along this direction. Moreover, the expression $\left.\bar{V}_{k G}^{\mathbf{i} E *} V_{k G}^{\mathbf{j}} / \epsilon_{F}-E_{f}\right)$ which determines in this case the mixing integrals $\mathcal{B}_{\mathbf{i j}}^{G E}$ turns into zero for $\mathbf{k}=[1,0,0]$ due to the similar reasons. Thus one can expect that the main characteristic features of the interplay between the HF and CF states, including the pseudogap, would develope in the $b c$-plane of the Brilloun zone. However, this kind of theory has an undesirable consequence: in the mean-field slave-boson approximation the spin and charge pseudogaps simply coinside, and this statement obviously contradicts the experimental data for CeNiSn.

Another version of the mean-field description of pseudogap in CeNiSn was presented recently in [24]. It was postulated in this theory that the ground state of Ce ion is the doublet $|\mp 3 / 2\rangle$, and the CF excitations where not taken into account. The pseudogap in the HF spectrum appeared due to the mentioned non-bonding character of f-f overlap matrix element $\mathcal{T}^{G G}(\mathbf{k})$. Then the low-temperature behavior of specific heat and magnetic susceptibility are determined by this pseudogap, and the satisfactory agreement between the theoretical description of these quantities and the experimental data for CeiSn can be achieved.

As to the inelastic neutron scattering cross section, the theoretical results presented in [24] are too scanty to conclude about agreement between the theory and the experiment. One can definitely mention the qualitative agreement, at least for the constant-E scans (figure 11 of Ref. 24] which is more or less similar to our figure 8). As to the constant-Q scans, the slaveboson description is able to describe the pseudogap in the two-particle HF DOS (figure 10 of Ref. 24]), although the experimental picture is, apparently, much more reach then the smooth curves presented in that figure. One should mention also, that the theoretical spectrum for $\mathbf{Q}=[1 / 2,0,0]$ presented in figure $10 \mathrm{~b}$ demonstrates the peak in a-direction which is absent in experiment because of the geometrical factor entering the neutron scattering cross section for this direction [7, 97. It is unclear also whether this theory is able to describe the quasi $1 \mathrm{D}$ character of neutron scattering along the $\left[0, n+1 / 2, Q_{c}\right]$ direction. On the face of it, this task seems to be rather difficult since the matrix element which governs the HF dispersion law behaves as $V^{2}\left(\hat{k}_{x}^{2}+\hat{k}_{y}^{2}\right)\left(1+15 \hat{k}_{z}^{2}\right.$ ) (see equation 2.10b in Ref. 24]). Besides, the CF level scheme adopted in this model is not supported by the experimental data available [ [ coincidence of the charge and spin pseudogap in the mean field slave boson theory mentioned above does not allow to reproduce the metallic charater of low-temperature resistivity. The absence of $T^{2}$ in the theoretical curve $\rho(T)$ is clearly seen from figure 13 of Ref. 24.

The inelastic neutron scattering method is an adequate tool for observing the spin-liquid excitations in the heavy-fermion materials. These measurements give the detailed information concerning the density of states of spinon particle-hole excitation spectrum, and the dispersion of various branches of spinon excitations can be restored by analysing various constant- $\mathbf{Q}$ and constant- $E$ scans. The general picture of low-energy excitation spectrum obtained from this analysis allowed us to describe also the low-temperature specific heat of CeNiSn (see also [25]). 
All other important characteristics of this material, such as magnetic susceptibility, thermal expansion, NMR relaxation rate, etc, also can be described quantitatively within the same approach.

The specific features of spin-liquid excitation spectrum do not violate the conduction electron spectrum which remains essentially metallic, although the anisotropy of spin excitations can result in anisotropy of scattering mechanism for conduction electrons, and the soft crystal field

excitations can influence their paramagnetic response. In any case, CeNiSn and related materials should be treated as metallic Kondo lattices with specific type of spin excitations.

\section{Acknowledgements}

We are indebted to H. Kadowaki, S. Kambe, and T. Sato for timely providing us with experimental information and important comments, to N.V.Prokof'ev for valuable critical remarks and to A.Yu.Yaresko for assistance in numerical computations. The support of the grants NWO 5-16501, INTAS 93-2834 and RFBR-95-02-04250 is acknowledged. 


\section{A Appendix}

To calculate the anisotropy factor in Cornut-Coqblin Hamiltonian one should expand the Bloch waves entering the RKKY interaction (11),(13) in partial waves entering the hybridization integrals in equation (5) and then superimpose the direction connecting two ions with the quantization axis by means of the matrices of finite rotations.

In the case of short enough interionic distances one can neglect the influence of angular dependence of the partial waves on the values of radial overlap integrals. This approximation [14. in a simplest case of the doublet formed by the $j=5 / 2$ states with the same $j_{z}$ value, i.e., $|\Gamma \nu\rangle=|j \pm M\rangle$ results in the following equation for the factor of anisotropy of exchange interaction,

$$
\begin{gathered}
B_{G}(\theta)=\left(B_{M}(\theta)\right)^{2}, \\
B_{M}(\theta)=\sum_{l=0,2,4,6}(-1)^{l / 2}(2 l+1) Z_{l}^{M} P_{l}(\cos \theta), \\
Z_{l}^{M}=\xi_{M}^{2} O(l, M+1 / 2)+\eta_{M}^{2} O(l, M-1 / 2), \\
\xi_{M}=\sqrt{(7+2 M) / 14}, \quad \eta_{M}=\sqrt{(7-2 M) / 14}, O(l, j)=C_{30 l 0}^{30} C_{3 j l 0}^{3 j}
\end{gathered}
$$

Here $C_{3 M l m}^{3 M^{\prime}}$ are the Clebsch-Gordan coefficients. This equation can be found in [14] for the case of $\theta=0$. Since the $\mathrm{CF}$ wave functions transform in accordance with the spherical group the only source of anizotropy is the definite direction of quantatization axis which lowers symmetry to the axial one. Therefore the interaction depends on the polar angle $\theta$ only.

More complicate is the anisotropy factor for a mixed representations of the type of eq. (27). In this case the wave functions transform according to the irreducible representation of the trigonal group with third order rotational axis parallel to the quantization axis. In this case the RKKY intraction becomes $\phi$-dependent,

$$
\begin{aligned}
B_{G}(\theta, \phi)= & \left\{1+\frac{2}{7}\left(5-9 a^{2}\right) P_{2}(\cos \theta)+\frac{3}{7}\left(1+a^{2}\right) P_{4}(\cos \theta)\right. \\
& \left.-\frac{a \sqrt{1-a^{2}}}{7 \sqrt{10}} P_{4}^{3}(\cos \theta) \cos (3 \phi)\right\}^{2}
\end{aligned}
$$

For the specific cases of interaction "in plane" $(\theta=\pi / 2)$ and along the quantization axis $(\theta=0)$ the factor $B$ does not depend on the angle $\phi$. Therefore the ratio between the "in plane" interaction and interaction along z-axis can be expressed in terms of single parameter $a$ entering the wave function (27),

$$
\frac{B_{G}(\theta=\pi / 2)}{B_{G}(\theta=0)}=\left\{\frac{1}{40} \frac{25+81 a^{2}}{4-3 a^{2}}\right\}^{2}
$$

It is seen from (58) that $B_{G}(\theta=\pi / 2) / B_{G}(\theta=0) \gg 1$ only when $|1 / 2\rangle$-compomemt of the crystal field wave function (27) is dominant.

In the case of the large interionic distances the asymptotic behavior of RKKY exchange integrals is dominated by $j_{z}= \pm 1 / 2$ partial waves [26], and the "in plane" interaction is stronger than that along the quantization axis $(\theta=0)$ in the case when the $|5 / 2\rangle$-component of the crystal field wave function (27) is dominant. 


\section{Figure captions}

Figure 1: The $b c$ plain of CeNiSn lattice. Two Ce sublattices are denoted by black and grey circles, resp. The orthorhombic distortion $\mathrm{O}$ (solid arrows) transforms the simple hexagonal lattice into two-sublattice orthorhombic one. The vector $\mathbf{d}$ is the basis vector of two-ion elementary cell

Figure 2: The spinon dispersion in CeNiSn along the high symmetry directions of 2D Brillouin zone obtained for the following paramenter values (in Kelvins) of the secular matrix (37): $\mathcal{T}_{1}=18.0, \mathcal{T}_{2}=12.7, \widetilde{\Delta}_{C F}=13.5, \mathcal{G}_{1}=3.5, \mathcal{G}_{2}=7.7$. Horizontal line at the energy $E=-13 \mathrm{~K}$ is the Fermi level position at $T=0$. The arrows indicate the intraband and interband transitions responsible for the main peaks in neutron scattering cross section (see text).

Insert: the non-hybridized spinon spectrum with CF level (dased line) superimposed

Figure 3: Upper panel: the density of states of unhybridized spinon spectrum

Lower panel: the density of states for spin-fermions hybridized with CF excitation. All other designations are the same as in fig. 2

Figure 4: The $\mathbf{Q}$ vectors corresponding to the main transitions in spinon bands in 2D reciprocal lattice. The shaded areas are the projection of BZ onto bc plain and the regions in BZ where the final states are located

Figure 5: Comparison of experimantal (diamonds) and theoretical (curves) constant- $\mathbf{Q}$ scans. Upper panel: $\mathbf{Q}=(0,0,1.2)$. Experimental poins are taken from [7].

Lower panel: $\mathbf{Q}=(0,1,0)$. Experimental points are taken from [8, 9]

Figure 6: Theoretical (lines) and experimental (points) constant-Q scans with $4 \mathrm{meV}$ peak. Upper panel: Constant- $\mathbf{Q}$ scan for $\mathbf{Q}=(0,1.5,1)$. Experimental points are taken from [8, 9] (squares) and [10] (diamonds).

Lower panel: Constant- $\mathbf{Q}$ scans for $\mathbf{Q}=(0,1.5,1)$ (solid line), $\mathbf{Q}=(0,1.5,0.75)$ (dotted line) and $\mathbf{Q}=(0,1.5,0.5)$ (dashed line)

Figure 7: Constant- $\mathbf{Q}$ scans for: $\mathbf{Q}=(0,1.5,0) ;(0,1.4,0) ;(0,1.3,0) ;(0,1.2,0) ;(0,1.1,0) ;(0,1,0)$ (curves $1-6$, respectively). Position of $4 \mathrm{meV}$ and $2 \mathrm{meV}$ peaks are indicated by arrows. The vertical scales are shifted for clarity

Figure 8: Theoretical (curves) and experimental (dots) constant-E scans $(E=4.2 \mathrm{meV})$ along $\mathbf{Q}=\left(0, Q_{b}, 0\right)$ (squares and solid line) and $\mathbf{Q}=\left(0, Q_{b}, 1\right)$ (diamonds and dashed line) directions. Experimental points are taken from [9]

Figure 9: Theoretical (curves) and experimental (dots) constant-E scans along $\left(0, Q_{b}, 1\right)$ direction: (a) $\mathrm{E}=4.2 \mathrm{mV}$; (b) $\mathrm{E}=3.3 \mathrm{meV}$; (c) $\mathrm{E}=2.5 \mathrm{meV}$. Experimental points are taken from 10

Figure 10: Constant- $\mathbf{Q}$ scans for: $\mathbf{Q}=(0,1.25,1)$ (dashed line); $\mathbf{Q}=(0,1.5,1)$ (solid line); $\mathbf{Q}=(0,1.75,1)$ (dotted line).

Figure 11: Theoretical (curves) and experimental (dots) constant-E scans $(E=4.25 \mathrm{meV})$ along $\mathbf{Q}=\left(Q_{a}, 3 / 2,0\right)$ direction. Experimental points are taken from [9] 
Figure 12: Theoretical curve for low- $T$ contribution to the specific heat in comparison with the experimental data taken from [5] (diamonds). The constant electronic contribution $\gamma_{e l}=18 \mathrm{~mJ} / \mathrm{mol} \mathrm{K}{ }^{2}$ is added to the theoretical curve (see text) 


\section{References}

[1] P. Fulde, Electron Correlations in Molecules and Solids, 3rd. Edition (Springer, Berlin, 1995) Chapt. 13.

[2] Yu. Kagan, K.A. Kikoin, and N.V. Prokof'ev, JETP. Lett. 57, 600 (1993)

[3] T. Takabatake and H. Fujii. Jpn. J. Appl. Phys. Ser.8, 254 (1993)

[4] P.A. Alekseev, V.N. Lazukov, E.S. Clementyev, E.V. Nefedova, I.P. Sadikov, M. N. Chlopkin, A.Yu. Muzhicka, I.L. Sashin, N.N. Efremova and V. Bührer, JETP, 79, 665 (1994)

[5] T. Takabatake, G. Nakamoto, T. Yoshino, H. Fujii, K. Izawa, S. Nishigori, H. Goshima, T. Suzuki, T. Fujita, K. Maezawa, T. Hiraoka, Y. Okayama, I. Oguro, A.A. Menovsky, K. Neumaier, A. Brückl, and K. Andres., Physica B 223\&224, 413 (1996)

[6] K. Nakamura, Y. Kitaoka, K. Asayama, T. Takabatake, G. Nakamoto, H. Tanaka, and H. Fujii. Physica B206\&207, 829 (1995)

[7] T.E. Mason, G. Aeppli, A.P. Ramirez, K.N. Clausen, C. Broholm C, N. Stücheli, E. Bucher, and T.M.M. Palstra, Phys. Rev. Lett. 69490 (1992)

[8] H. Kadowaki, T. Sato, H. Yoshizawa, T. Ekino, T. Takabatake, H. Fujii, L.P. Regnault, and Y. Ishikawa, J. Phys. Soc. Jpn, 63, 2074 (1994)

[9] T. Sato, H. Kadowaki, H. Yoshizawa, T. Ekino, T. Takabatake, H. Fujii, L.P. Regnault, and Y. Ishikawa, J. Phys.: Cond. Mat. 7, 8009 (1995)

[10] S. Kambe, S. Raymond, H. Suderow, J. McDonough, B. Fak, L.P. Regnault, and J. Flouquet, Physica B 223\&224, 135 (1996)

[11] K.A. Kikoin, A. de Visser, K. Bakker, and T. Takabatake, Z. Phys. B94, 79 (1994)

[12] G. Baskaran, Z. Zou, and P. W. Anderson, Solid State Comm. 63, 973. (1987).

[13] K.A. Kikoin, M.N. Kiselev, and A.S. Mishchenko, JETP Letters 60, 600 (1994); Physica B206\&207, 129 (1995)

[14] B.Coqblin and J.R.Schrieffer, Phys.Rev., 185, 847 (1969)

[15] B.Cornut and B.Coqblin, Phys.Rev.B, 5, 4541 (1972)

[16] Yu. Kagan, K.A. Kikoin, and N.V. Prokof'ev, Physica B 182, 201 (1992)

[17] I. Higashi I, K. Kobayashi, T. Takabatake, and M. Kasaya, J. Alloys and Compounds 193, 300 (1993).

[18] S. Doniach, Proc. Phys. Soc., 91, 86 (1967).

[19] S. Nohara, H. Namatame, F. Fujimori, and T. Takabatake, Phys. Rev.B 47, 1754 (1993)

[20] A.J. Nolten, M. Sci Thesis, Amsterdam University, Amsterdam, 1994. 
[21] A.J. Nolten, A. de Visser, F.E. Kayzel, J.J.M. Franse, H. Tanaka, and T. Takabatake, Physica B 206\&207, 825 (1995).

[22] K.A. Kikoin, J. Phys.: Cond. Matt. 8, 3601 (1996).

[23] K. Hanzawa, K. Yamada and K. Yosida, J. Phys. Soc. Jpn 56, 678 (1987).

[24] H.Ikeda and K. Miyake, J. Phys. Soc. Jpn 65, 1769 (1996).

[25] K.A. Kikoin, M.N. Kiselev, and A.S. Mishchenko, Czech. J. Phys., 46, Suppl. S4, 1899 (1996).

[26] B.R. Cooper, R. Siemann, D. Yang, P. Thayamballi, and A. Banerjea, in The Handbook on the Physics and Chemistry of Actinides, edited by A.J.Freeman and G.H.Lander (NorthHolland, Amsterdam, 1985), Vol. 2, Chap. 6, pp. 435-500. 


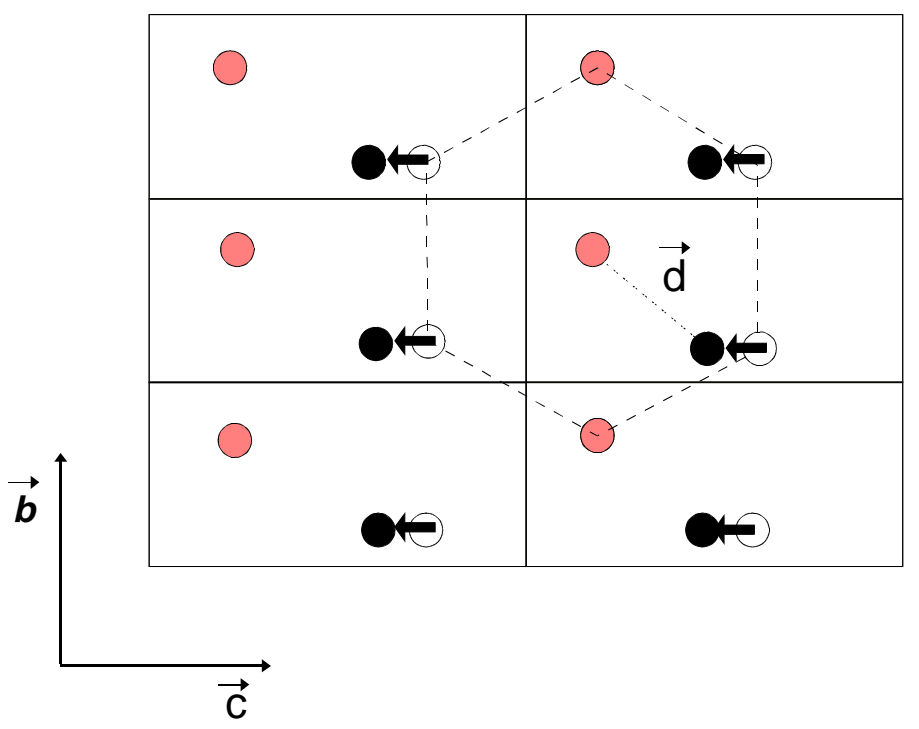

Fig. 1. 


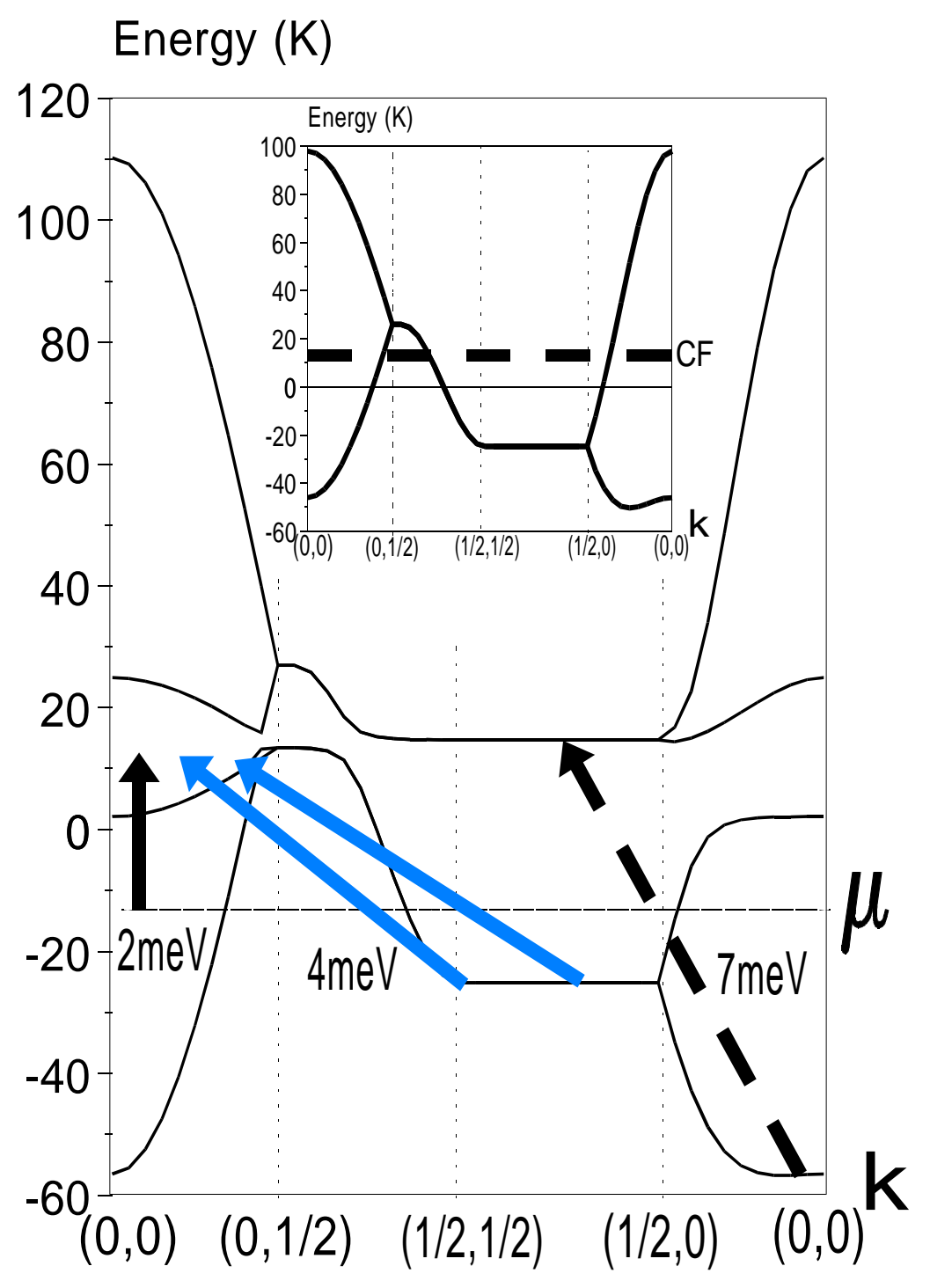

Fig. 2. 

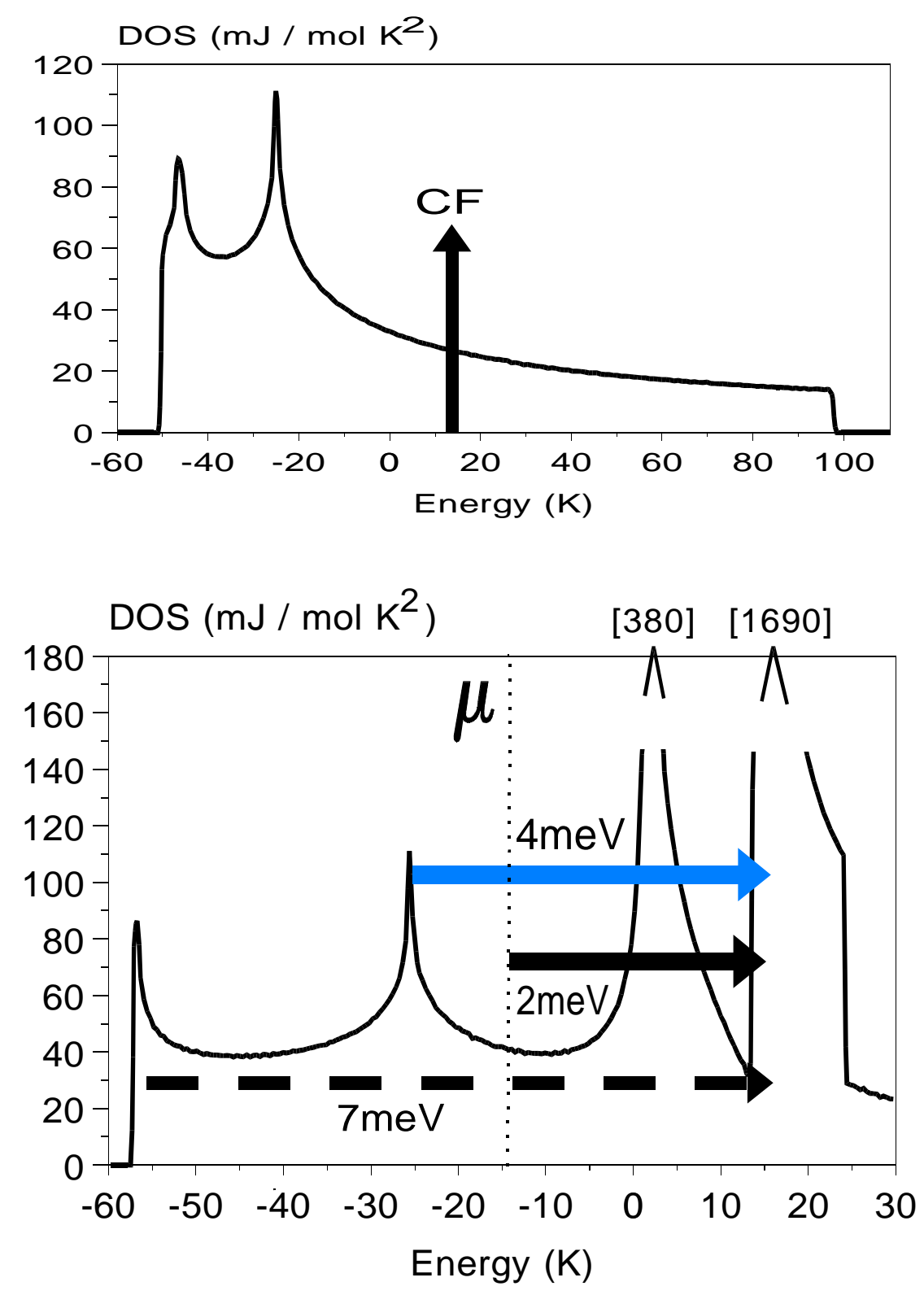

Fig. 3. 


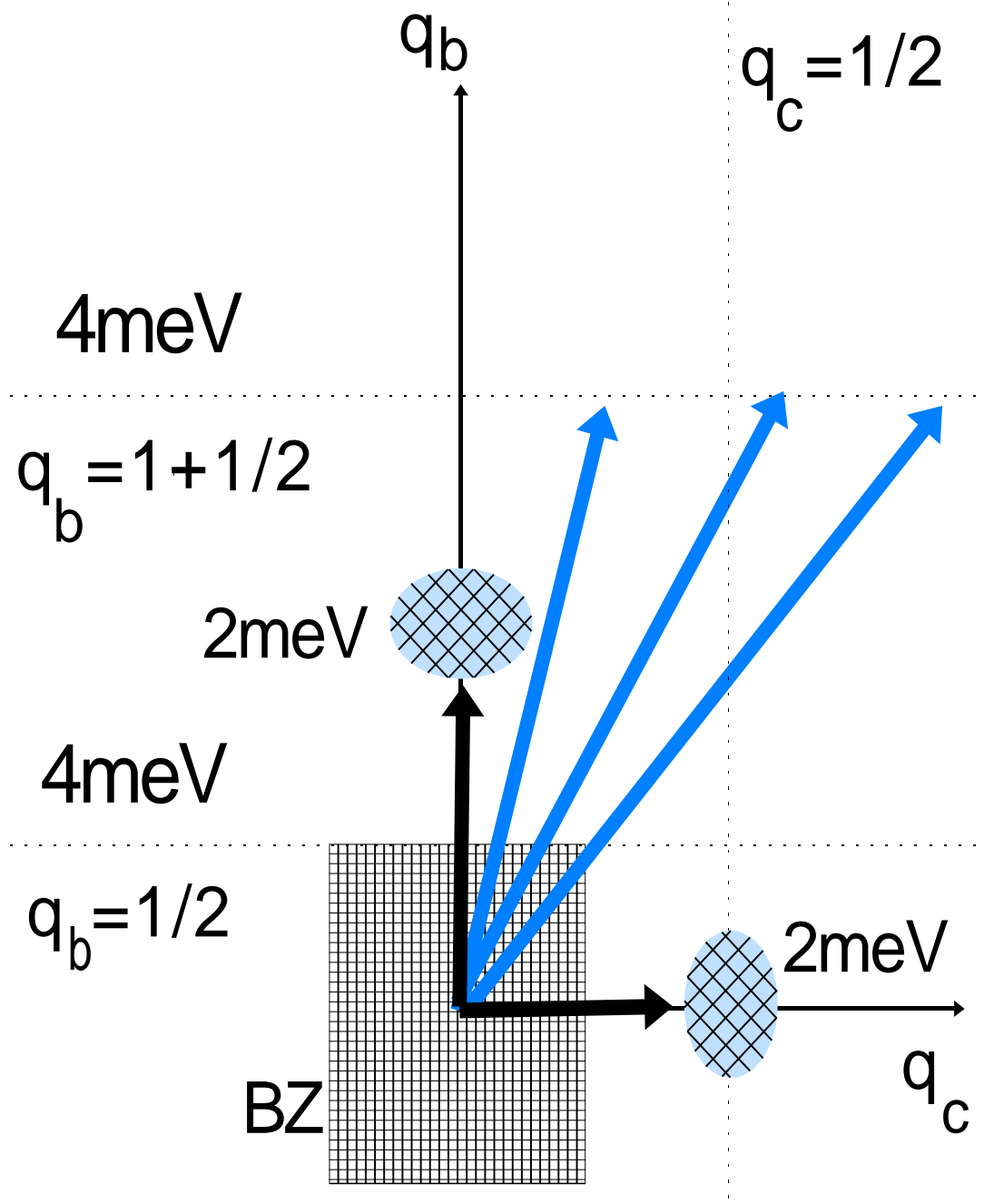

Fig. 4. 
Counts
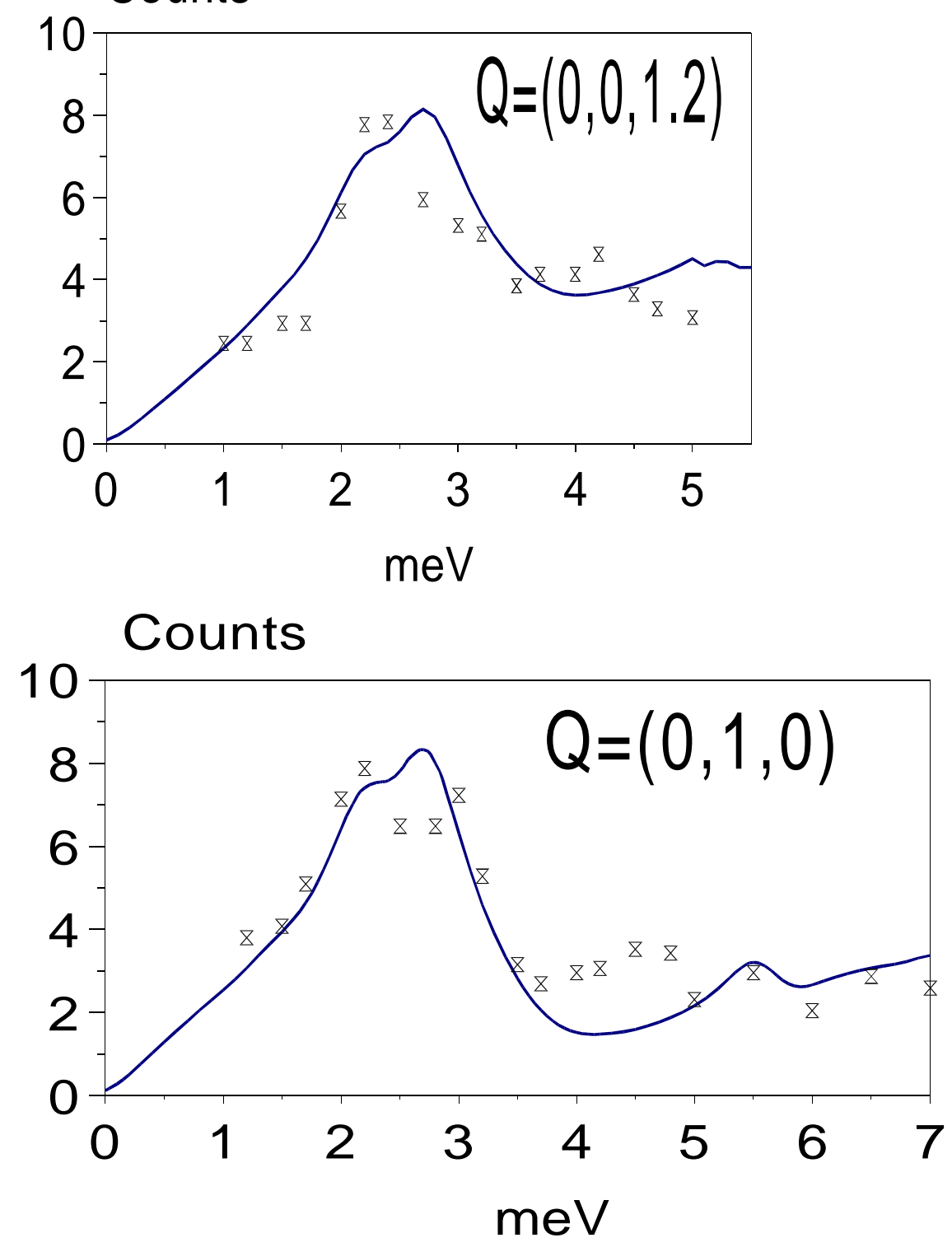

Fig. 5. 

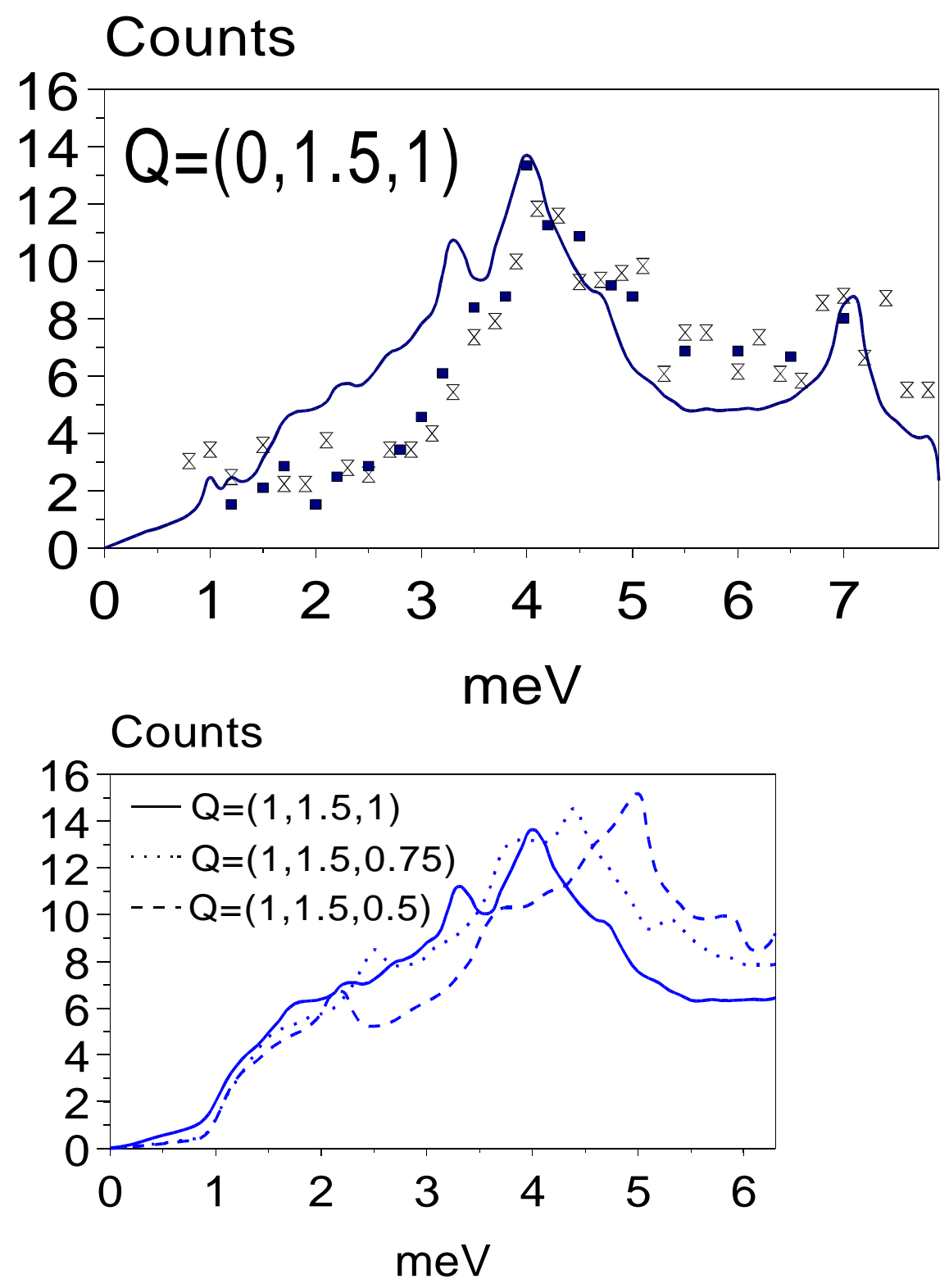

Fig. 6 . 
Counts (A.U.)

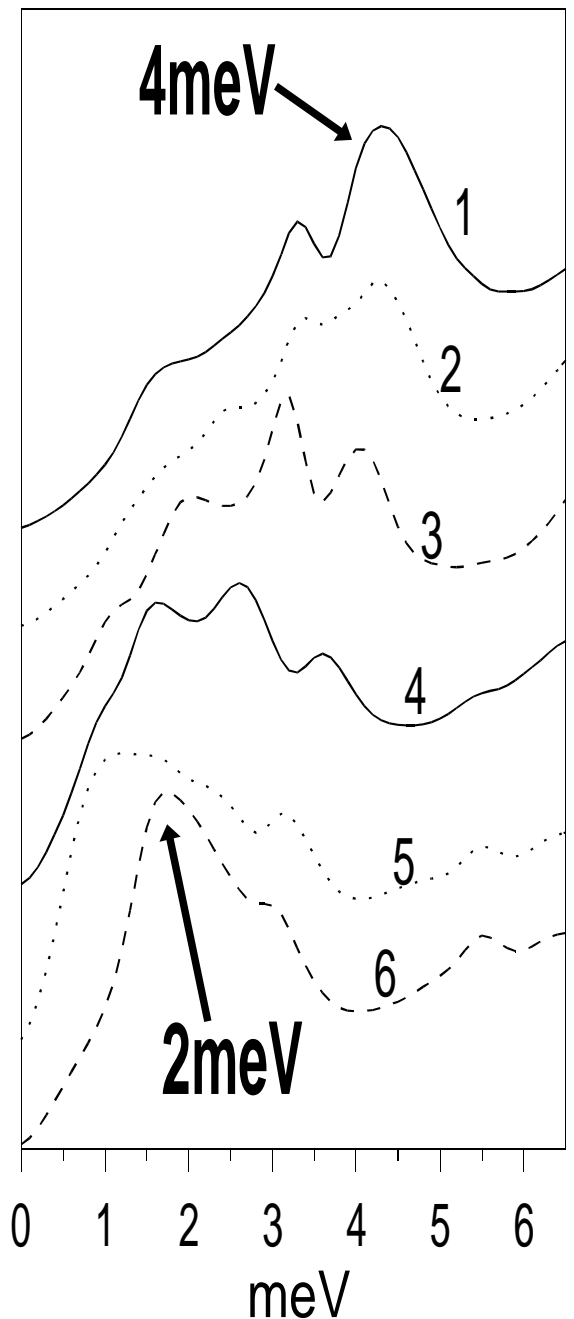

Fig. 7. 


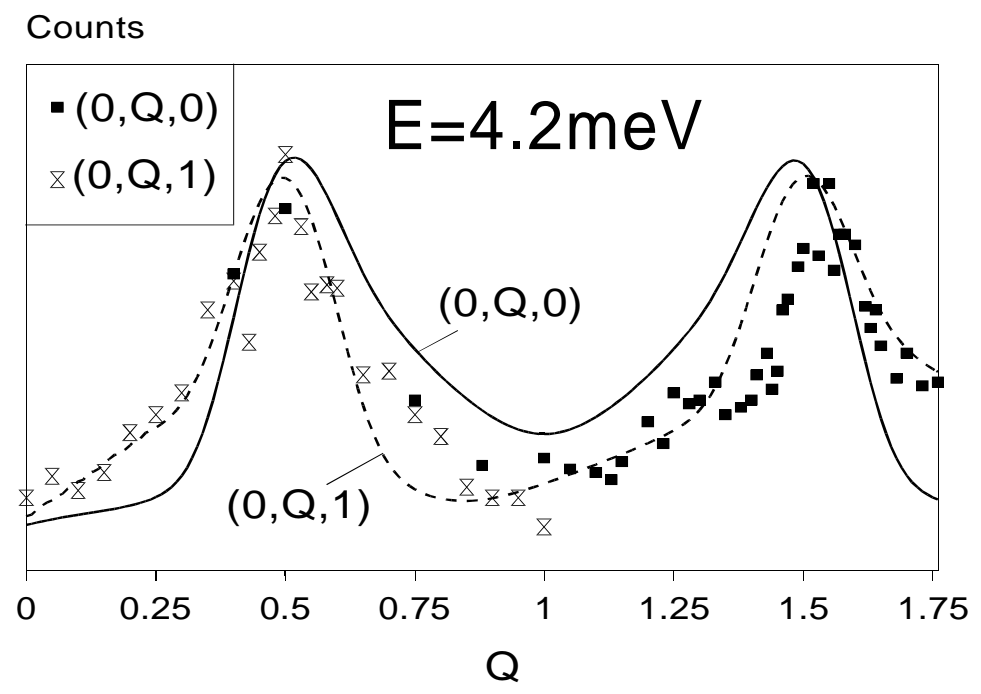

Fig. 8. 
Counts (A.U.)

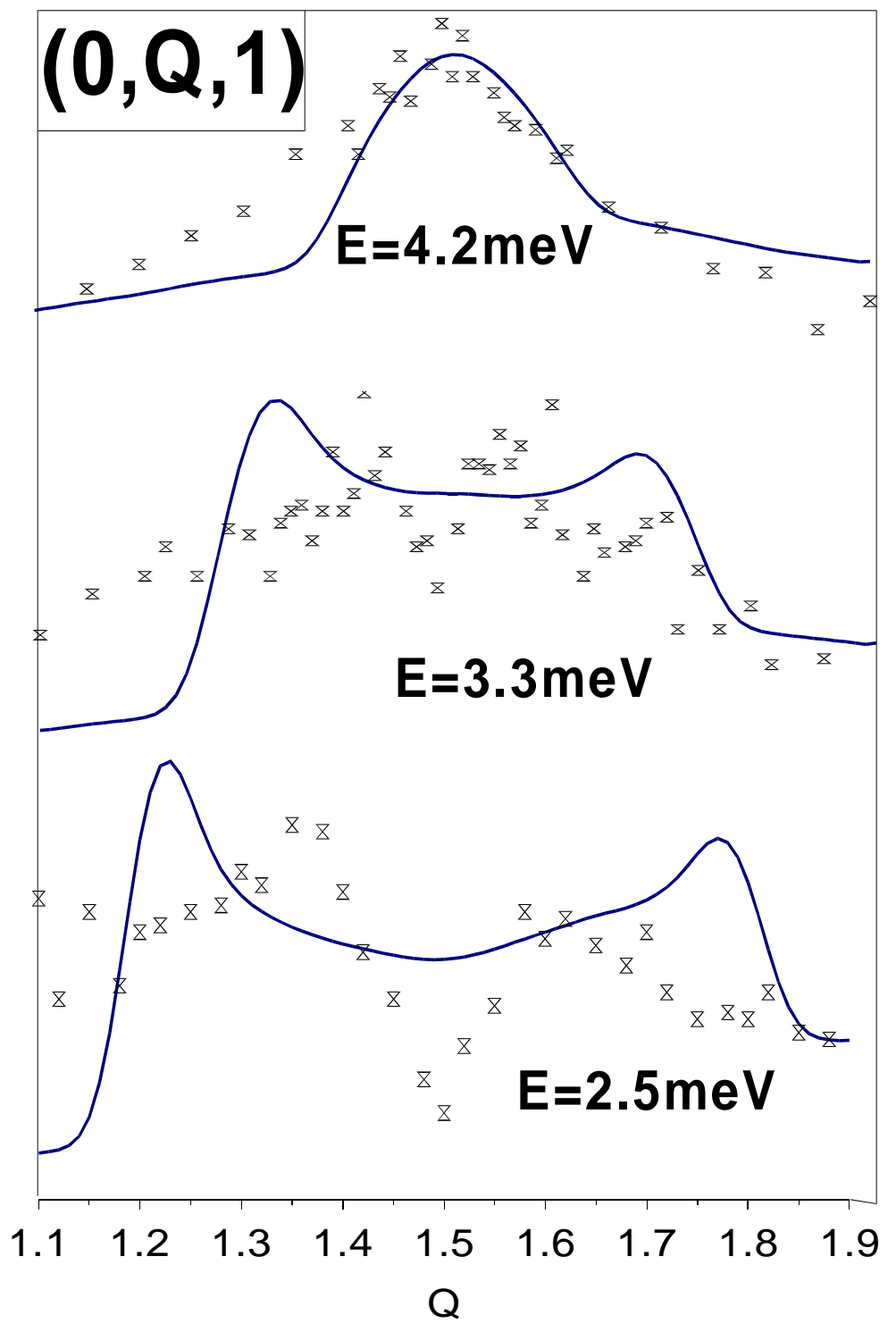

Fig. 9. 


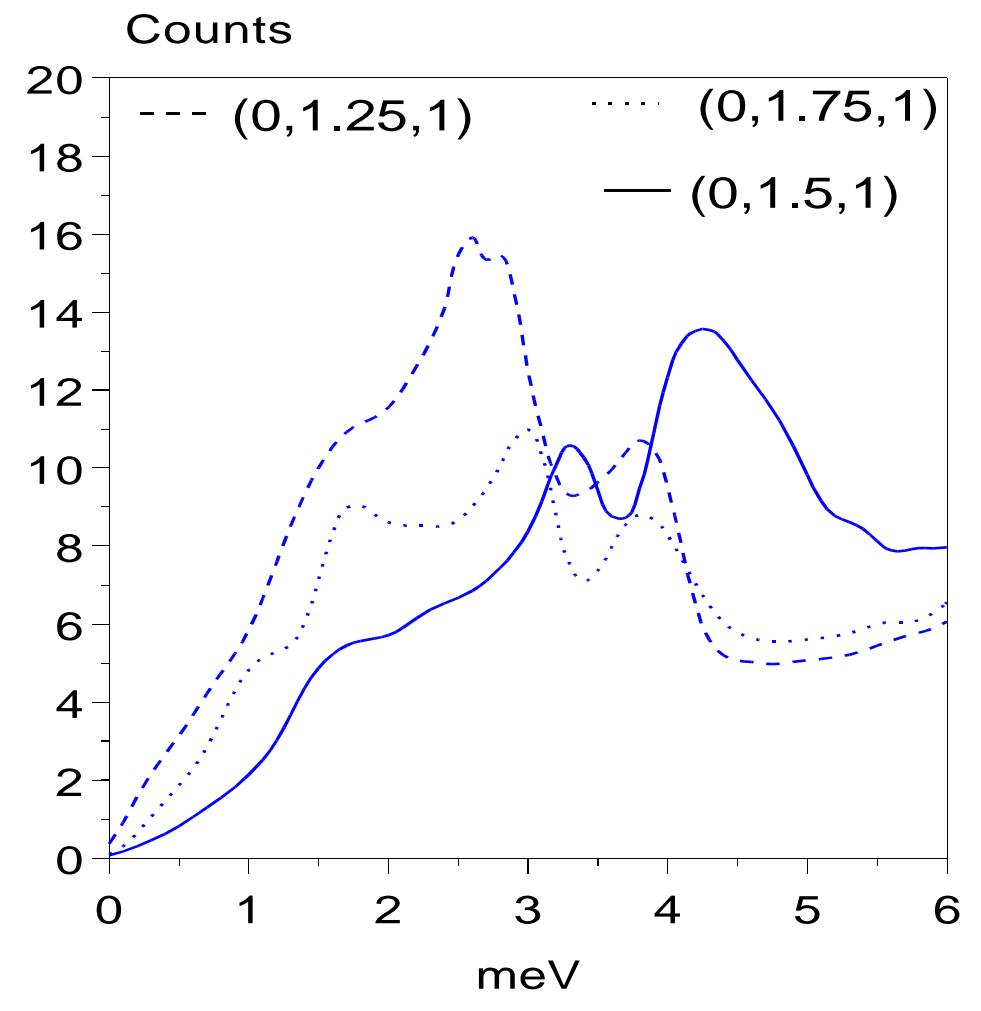

Fig. 10. 
Counts (a.u.)

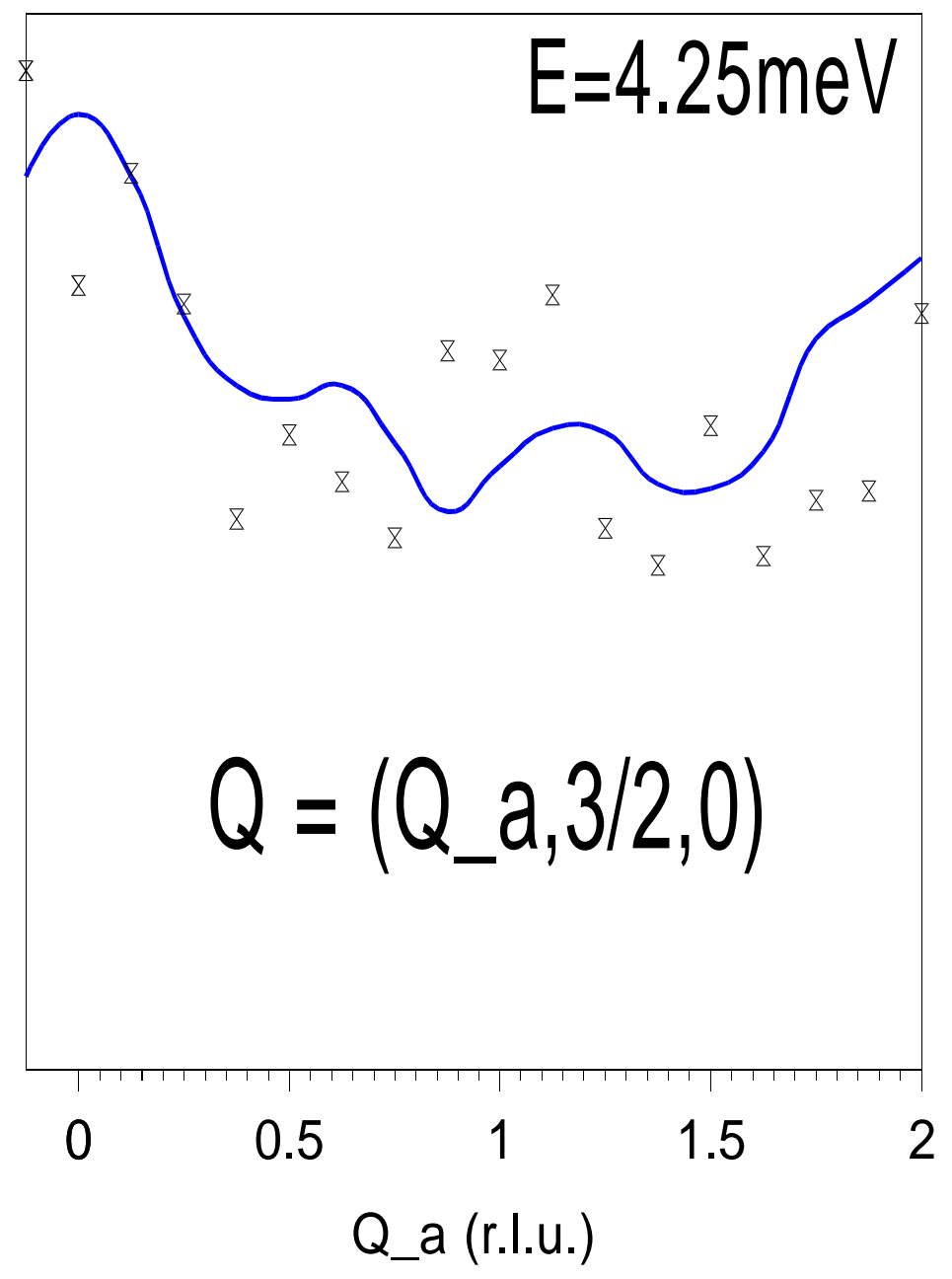

Fig. 11. 


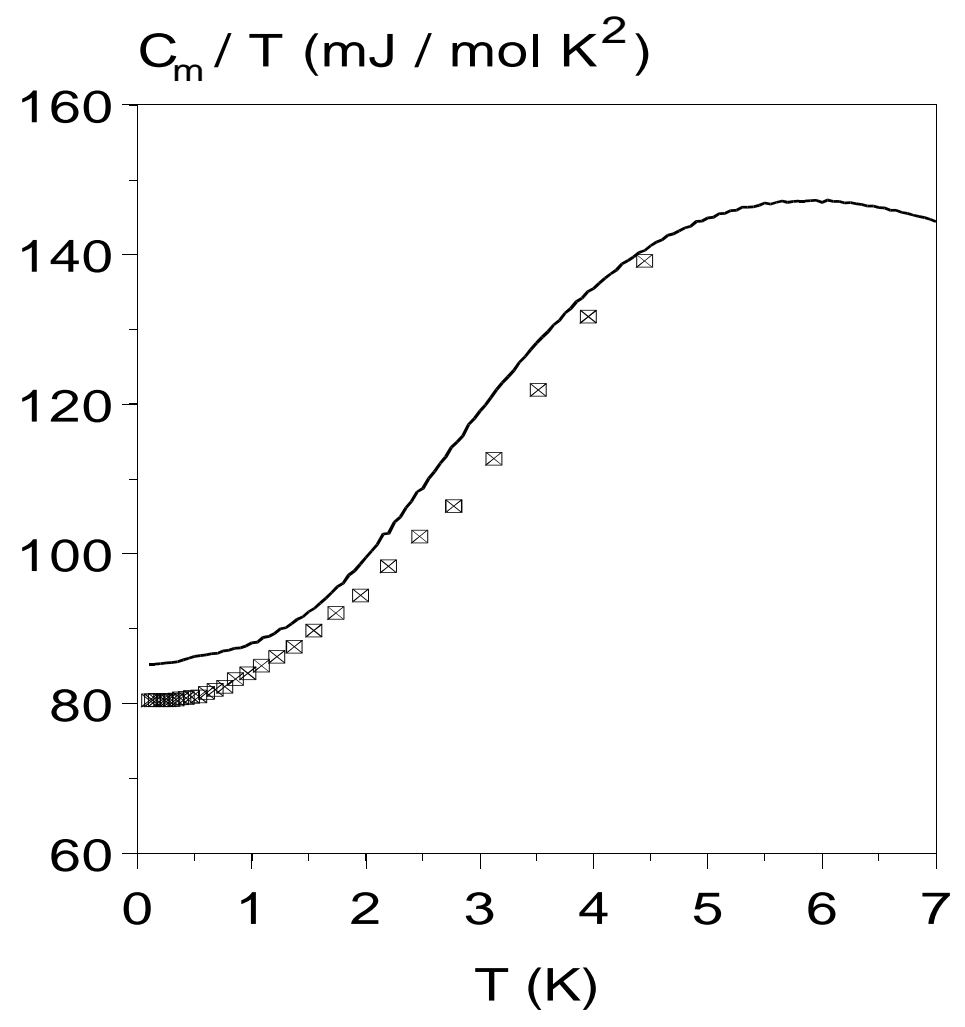

Fig. 12. 